\title{
Estructura y organización de los ensambles de roedores de un bosque de la Sierra Madre Occidental, Durango, México
}

\author{
Structure and organization of rodent assembles of a forest of the Sierra Madre Occidental, \\ Durango, Mexico
}

ELIZABETH E. ARAGÓN ${ }^{1, *}$, ALFREDO GARZA ${ }^{1}$ \& FERNANDO A. CERVANTES ${ }^{2}$

\author{
${ }^{1}$ Centro de Ecología Regional, A.C. Blvd. Durango 501, Col. Valle del Sur. Durango 34120 Dgo, México \\ ${ }^{2}$ Instituto de Biología, UNAM. Departamento de Zoología, UNAM. Apartado Postal 70-153, C.P. 04510, Coyoacán, \\ México, D.F. \\ *Autor correspondiente: eeapliz@hotmail.com
}

\begin{abstract}
RESUMEN
La diversidad biológica de los bosques templados en la región de montaña denominada "Sierra Madre Occidental», México, es rica. Esta zona se encuentra en la Zona de Transición, un área biogeográficamente importante de Norteamérica caracterizada por una gran heterogeneidad ambiental local. Sin embargo, poco se conoce sobre la composición taxonómica y la organización de los mamíferos pequeños nativos y sobre la influencia de los factores ecológicos locales, en particular en tipos de vegetación diferentes. Por lo tanto, se estudió la composición de especies y la estructura de los ensambles de pequeños mamíferos de esta sierra y se proporcionó información sobre el nivel de anidamiento y la detección de patrones en su organización. El área de estudio fue el rancho "El Durangueño", Durango, México. Se muestrearon tres tipos de vegetación diferentes (pastizales y bosques de encino-pino y pino-encino) por estación de 2005 y 2006. Los roedores fueron capturados, recapturados e identificados durante tres noches por sitio. Los resultados produjeron 520 individuos (386 capturas, 134 recapturas) de 18 especies de mamíferos pequeños (14 ratones, cuatro ardillas) de tamaños corporales diferentes, de afinidades neártica, neotropical o compartida; se encontraron endemismos y taxas en alguna categoría de riesgo de extinción. La riqueza y la equitabilidad fueron altas, la diversidad y los niveles poblacionales fueron moderados, a excepción de un bosque de encino-pino con densidad de pequeños mamíferos alta y la dominancia de alguna especie en el bosque de pino-encino, debido probablemente a perturbaciones. El patrón espacial de las especie de tamaño mediano no fue azaroso. El ensamblaje de especies no presentó anidamiento y tuvo una distribución log-normal, con cuatro especies comunes y de amplia distribución (Peromyscus truei, P. maniculatus, Reithrodontomys montanus y Sigmodon leucotis) y siete de distribución restringida. Las especies pertenecieron a gremios tróficos diferentes, como lo muestran sus preferencias alimentarias, que estuvieron compuestas por hasta dos especies dominantes por sitio. En conclusión el ensamble de mamíferos pequeños de estos bosques de la Madre Sierra es distintivo y diverso, no está estructurado al azar y la vegetación puede ser uno de los factores ecológicos que han jugado un papel evolutivo importante en su formación.
\end{abstract}

Palabras clave: bosque templado, conservación, ensamble, roedor, Sierra Madre Occidental.

\begin{abstract}
Biodiversity of temperate forests in the mountain region called "Sierra Madre", at western Mexico, is rich. This zone lies in the Transition Zone, a biogeographical important area of North America characterized by a remarkable local environmental heterogeneity. However, little is known about the taxonomic composition, and organization of the assemblage of native small mammal, and the influence of local ecological factors, particularly from different vegetation types. Therefore, we were interested in documenting the species composition and structure of the small mammal assemblages of this sierra and provide insights into the nesting level of the community and deviations from a random organization. The study area was a place named "El Durangueño" ranch, Durango, México. Three different vegetation types (grassland, and oak-pine and pine-oak forests) were sampled there during every season of 2005 to 2006. During three consecutive nights rodents of each site were captured, recaptured, and taxonomically identified. Results produced 520
\end{abstract}


individuals (386 captures, 134 recaptures) of 18 small mammal species (14 mice, four squirrels) of different body sizes that represent neartic, neotropical, or shared affinity; endemicity of taxa was found as well as taxa in some extinction risk category. Species richness and eveness were high and the diversity and population levels were moderate, although high density of small mammals highlights oak-pine forest whereas dominance of some species occurs in pine-oak forest perhaps due to disturbance. Spatial pattern of medium-size species was not at random. Assemble of species revealed a non-nested pattern and displayed a log-normal distribution, characterized by four common and widely distributed rodent species (Peromyscus truei, P. maniculatus, Reithrodontomys montanus, and Sigmodon leucotis), and seven of restricted distribution. On the other hand, several species were allocated to different trophic-niche guilds, as revealed by their reported food preferences, that were composed by up to two dominant species by site. In conclusion, the small mammal assemblage of forests of Sierra Madre is distinctive and diverse, is not structured at random and vegetation may be one of the ecological factors that have played an important evolutionary role.

Key words: assemble, conservation, rodent, Sierra Madre Occidental, temperate forest.

\section{INTRODUCCIÓN}

La Sierra Madre Occidental de México (SMOcc) constituye una provincia fisiográfica con una gran superficie, siendo el $30 \%$ del territorio nacional y la de mayor extensión para el estado de Durango, por lo que es el primer estado forestal a nivel nacional (SEMARNAT 2009, http://www.semarnat.gob.mx/estados/ durango/temas/Paginas/aprov_forestal.aspx). Esta región tiene una alta heterogeneidad ambiental, por presentar una variabilidad fisiográfica y climática diversa, que van desde sitios fríos en las cimas, los húmedos en la región suroeste, los semisecos en las partes norte y noreste (Challenger 1998), hasta los subtropicales. Estos climas son influenciados por la pendiente y la orientación de las laderas, el tipo y la profundidad del suelo. Estos atributos y la compleja historia biogeográfica (eventos del Plioceno y Pleistoceno, Challenger 1998) han dado lugar a distintas comunidades bióticas y mosaicos de vegetación en áreas relativamente pequeñas y cercanas, con una topografía accidentada.

Se ha sugerido que la SMOcc funciona como corredor biológico, que combina elementos neárticos y neotropicales facilitando la presencia de endemismos por aislamiento geográfico, en donde se favorece la especiación de plantas y animales (González 1983, Fa \& Morales 1998, Ferrusquía-Villafranca 1998). Presenta una gran variedad de ambientes, resguardando una alta diversidad de mamíferos (104 especies), representando el $77 \%$ del total estatal (135 especies, Aragón et al. en prensa). Se estima que pueden existir gran número de endemismos de mamíferos (Ceballos \& Rodríguez 1993, Fa \& Morales 1998,
Challenger 1998), en su mayoría roedores y murciélagos. No obstante, hay pocos estudios sobre la biodiversidad en esta cadena montañosa y poco se sabe de los factores ecológicos que influyen en los ensambles de mamíferos.

Los pequeños mamíferos reportados para la SMOcc en Durango comprenden estudios realizados al sureste del estado por Muñiz (1988) con 16 especies, 13 múridos y tres sciúridos y en la Reserva de la Biosfera de La Michilía con 11 especies, un heterómido, nueve múridos y un sciúrido (Álvarez \& Polaco 1984, Sosa et al. 1995, Hernández 1996, Cuautle 2007). Mientras que para la SMOcc en Jalisco se han registrado 18 especies, tres heterómidos, 11 múridos y cuatro sciúridos (Guerrero et al. 1995, Iñiguez \& Santana 2005). Las investigaciones ecológicas son escasas, solo hay trabajos poblacionales de algunas especies comunes (Galindo \& Krebs 1997).

En otros bosques templados del país existe una alta riqueza de especies y densidad de roedores en altitudes intermedias (Galindo \& Krebs 1997, Brown 2001, Sánchez-Cordero 2001, Mena 2004). Patrón que ha sido observado en otras zonas montañosas del mundo (Heaney 2001, Md Nord 2001, Rickart 2001, McCain 2004) y que responden tanto a procesos históricos como ecológicos que operan a escala local y regional (Ricklefs \& Schluter 1993, Rickart 2001). También se conoce que la estructura del hábitat es determinante en los ciclos de vida de las especies e influyente en sus patrones de distribución y abundancia (Ceballos 1989, Galindo \& Krebs 1997). Por otra parte, las zonas de montaña son valiosas a nivel de conservación, pues concentran gran cantidad de 
endemismos (Patterson et al. 1998, Sánchez 2001).

Los bosques templados de Norteamérica (Canadá y Estados Unidos) representan zonas de alta productividad y diversidad biológica, debido a su alta heterogeneidad espacial (Canham et al. 1990, Lertzma et al. 1996, Tilman 1999), resultado de diferentes factores y procesos. Por lo anterior, se reporta que estos ambientes ofrecen una gran variedad de microhábitats y un intervalo amplio de microclimas, que se traduce en un mayor espectro de recursos (Kotler \& Brown 1988, Carey \& Johnson 1995, Carey et al. 1999). Esta información propone que los mamíferos pequeños pudieran ser indicadores de la condición de los bosques, dado que la heterogeneidad espacial promueve la diversificación de sus nichos espaciales, además de ser dispersores de semillas, esporas y propágulos de plantas, briofitas, hongos y líquenes, descomponedores de materia orgánica, reguladores de invertebrados y presas de gran número de vertebrados.

Para comprender la organización de los roedores de un sitio, además de describir la composición y estructura, una alternativa es la búsqueda de reglas de ensamblajes. Estas últimas son valiosas pues muestran patrones significativamente distintos al azar, de los cuales el uso de modelos nulos resulta ser una potente herramienta para estas valoraciones y actualmente es frecuentemente utilizada (Gotelli \& Graves 1996). En los bosques templados se pueden someter a prueba para roedores las reportadas en otras zonas (desiertos, bosques mesófilos o islas), para definir aspectos del ensamblaje de las especies: a) los de mayor riqueza de especies contengan a las de menor riqueza (anidación), por diferencias (movilidad y abundancia de los depredadores), limitaciones por condiciones abióticas y por interacciones negativas como la competencia (Diamond 1975, Patterson \& Brown 1991), que ocurre cuando se tiene una historia biogeográfica común, ambientes contemporáneos similares y organizaciones jerárquicas en las relaciones de nicho; b) las especies coexistan por diferencias morfológicas, como se ha reportado para el tamaño corporal y el peso (Brown \& Nicoletto 1991, Arita \& Figueroa 1999, Stone et al. 2000) $y, c)$ que los ensambles se estructuren por diferentes grupos funcionales (Fox 1987), que podrían conformarse en cuanto a gremios tróficos principalmente.

De acuerdo a lo anterior, el objetivo de este trabajo fue definir la composición y estructura a partir de la búsqueda de patrones de los ensambles de roedores en un bosque de la SMOcc, con el fin de conocer la organización de las mismas. Se parte de la hipótesis de que la composición de roedores esté integrada por especies de las zonas neártica y neotropical y que su estructura se caracterizará por presentar una organización no azarosa, con una alta riqueza y diversidad de roedores. Este conocimiento sentará las bases que permitan el desarrollo futuro de acciones de conservación, uso y manejo de estos recursos faunísticos dentro de los bosques templados del norte de México, escasamente estudiados.

\section{MÉTODOS}

El estudio se realizó en el rancho "El Durangueño" que está ubicado al noroeste del estado de Durango, México, en la Sierra Madre Occidental $\left(24^{\circ} 27^{\prime} 31^{\prime}\right.$ ' $\mathrm{N}$ y $\left.104^{\circ} 55^{\prime} 55^{\prime}, \mathrm{O}\right)$, con una altitud promedio de 2,500 metros sobre el nivel del mar. La topografía es accidentada, formada principalmente por lomeríos (pendientes de $25 \%$ a $35 \%$ ), mesetas altas (pendientes de hasta $10 \%$ ), cañadas con pendientes pronunciadas (45\%) y zonas escarpadas (10\% del predio).

El clima es semifrío con lluvias en verano y precipitaciones invernales entre 5 y $10.2 \%$ anual. La precipitación media anual varía de 800 a $1,000 \mathrm{~mm}$ y la temperatura promedio anual es de $5^{\circ}$ a $12^{\circ} \mathrm{C}$, con una estación seca de 6 a 7 meses de noviembre a mayo (García 1988).

Los tipos de vegetación predominantes en la SMOcc son el bosque de encino-pino y el de pino-encino en las partes más altas, principalmente en las laderas interiores de la SMOcc (González 1983). Prevalecen diversas especies de los géneros Pinus y Quercus, como otras arbóreas, el táscate (Juniperus deppeana Steud) y el madroño (Arbutus spp.), así como matorrales de manzanita (Arctostaphylos pungens Kunth) y diversas herbáceas y poáceas de los géneros Bouteloua, Mulhenbergia y Aristida (González et al. 1993, Aragón et al. en prensa). 
"El Durangueño" consta de 10,378 ha y la tenencia de la tierra es privada. Cuenta con 20 bordos de abrevadero y 16 manantiales de agua que son utilizados por la fauna silvestre. Las actividades productivas son ecoturismo, manejo y aprovechamiento cinegético de fauna silvestre y silvícola, escasa ganadería y agricultura de autoconsumo. Este estudio forma parte de un estudio sobre el monitoreo y protección de la biodiversidad del área (Garza et al. 2005).

El muestreo de las especies de roedores se realizó en nueve parcelas georreferenciadas de $70 \times 70 \mathrm{~m}^{2}$ durante las cuatro estaciones del año (2005-2006), en tres tipos de vegetación con tres réplicas: bosque de encino-pino, bosque de pino-encino y pastizales (Tabla 1).

En cada parcela se colocaron 64 trampas Sherman, con una distancia entre trampas de 10 $m$ durante tres noches, con una disposición de 8 filas por 8 columnas. Las trampas fueron cebadas con avena, esencia de vainilla y mantequilla de cacahuate.

Los animales capturados se determinaron mediante guías de campo, claves taxonómicas, experiencia personal y/o bajo la supervisión de taxónomos expertos (Hall 1981, Romero et al. 2000, Villa \& Cervantes 2003). Se utilizó la técnica de captura-marcaje-recaptura (Pielou 1974, Krebs 1999), liberando a los individuos en el sitio de su captura. Los datos registrados fueron: altitud, tipo de vegetación, especie, fecha, localidad, número de trampa, sexo, edad, peso, captura o recaptura y número de individuo. Se realizaron colectas científicas cuando la identificación de las especies en campo fue difícil, siendo catalogadas y depositadas en la Colección Nacional de Mamíferos del Instituto de Biología de la Universidad Nacional Autónoma de México.

\section{Análisis de la información}

Se realizaron comparaciones entre sitios y estaciones de los siguientes aspectos:

La estructura de tallas y pesos se obtuvo a partir de la distribución de frecuencia tanto de tallas como de pesos corporales de todas las especies observadas o capturadas en los sitios. Se consideraron los valores promedio de todos los individuos adultos de la misma especie. Las especies se ubicaron de acuerdo a su distribución en los siguientes biomas (WWF Nature Serve): Sierra Madre, Desierto, Grandes Planicies y de amplia distribución, de acuerdo a la sobreposición de las superficies de distribución de cada especie. Se realizó la prueba de la regla de Hutchinson (1959), dividiendo los valores más grandes de talla entre los más pequeños.

La composición taxonómica se determinó mediante la verificación de todas las especies en campo o en colecciones, la afinidad biogeográfica de cada especie a partir de las

TABLA 1

Ubicación geográfica de los sitios de muestreo en tres diferentes tipos de vegetación presentes en el rancho "El Durangueño", Municipio de Canatlán, Durango, México. Datum WGS83 Proyección de coordenadas Universal Transversa de Mercator (UTMs).

Geographical location of three sampling sites in the different types of vegetation in "El Durangueño" ranch, Municipio Canatlán, Durango, México. Datum WGS83 Universal Transverse projection of Mercator's coordinates (UTMs).

\begin{tabular}{lccccc}
\hline Cuadro & Vegetación & Nombre del Sitio & Coordenadas(UTM) & Altitud(m) \\
\hline 1 & Pastizal & Campo de Verano & 512318.98 & 2700871.98 & 2456 \\
2 & Pastizal & Fresada & 511327.88 & 2700769.29 & 2450 \\
3 & Pastizal & La Pista & 512175.69 & 2701476.19 & 2487 \\
4 & Bosque de encino-pino & El Aire & 511196.53 & 2699470.12 & 2515 \\
5 & Bosque de encino-pino & La Pila & 512340.47 & 2698455.14 & 2554 \\
6 & Bosque de encino-pino & Nogales & 511466.97 & 2702129.69 & 2486 \\
7 & Bosque de pino-encino & Casas & 513635.08 & 2697772.71 & 2496 \\
8 & Bosque de pino-encino & Kosovo & 510988.76 & 2699735.20 & 2513 \\
9 & Bosque de pino-encino & La Costosa & 511906.49 & 2697074.93 & 2501 \\
\hline
\end{tabular}


clasificaciones hechas por Villa \& Cervantes (2003), Ceballos \& Oliva (2005) e Iñiguez \& Santana (2005), así como el gremio trófico clasificando a las especies en grupos por la similitud en la alimentación, conforme a las categorías propuesta por Ceballos y Navarro (1991) y la información de las especies en México (Villa \& Cervantes 2003, Ceballos \& Oliva 2005).

Cada especie registrada se ubicó de acuerdo a su categoría de riesgo dentro de la Norma Oficial Mexicana vigente (NOM-059, SEMARNAT 2001). Las categorías de acuerdo a esta norma son: peligro de extinción (distribución o tamaño poblacional disminuido drásticamente y su viabilidad biológica natural en riesgo, que coincide parcialmente a la categoría de la IUCN), amenazadas (especies o poblaciones que podrían llegar a encontrarse en peligro de desaparecer a corto o mediano plazo, equivalente a vulnerable de la IUCN), sujetas a protección especial (especies o poblaciones que pueden llegar a encontrarse amenazadas por factores negativos a su viabilidad, requieren recuperarse y conservarse, incluyendo a las de menor riesgo para la IUCN)

La distribución de la abundancia relativa de especies en los diferentes sitios se evaluó mediante las tendencias observadas al graficar las abundancias acumuladas.

Se estimaron los parámetros del ensamble de roedores y su similitud entre los sitios de monitoreo. Para conocer la riqueza específica, diversidad y equitabilidad de los roedores en cada sitio (conteo de especies, índice de Shannon-Wiener H' e índice de Simpson, Magurran 1988), se utilizaron los programas Biodiversity Pro (ver. 2.0) y Past ver. (1.3), mientras que para estimar la abundancia relativa se utilizó el método del número mínimo de animales vivos o de enumeración (MNIV, Krebs 1966, 1999), extrapolando el valor obtenido a 1 ha. El peso total (gramos) por hectárea de los roedores se calculó por la suma de los pesos corporales de todas las capturas.

La información se analizó mediante estadística descriptiva. La variación entre los sitios de muestreo y las épocas se evaluaron mediante análisis de varianza (ANOVA, paramétrico) o de Kruskal-Wallis no paramétrico (Zar 1996). Se efectuó un análisis de agrupamiento de la abundancia de las especies de roedores, para determinar afinidad entre los sitios, utilizando el índice de similitud de distancias euclidianas y el método de agrupación de un solo enlace (linaje simple) mediante el programa Past ver. 1.3, Hammer et al., 2005).

El modelo nulo de coocurrencias de especies se utilizó para evaluar si el ensamble de roedores presenta una estructura azarosa o no, en cuanto a la presencia de las especies en los sitios (Stoner \& Robert 1990, Gotelli \& Graves 1996). Se hicieron matrices de presencia-ausencia de las especies agrupadas de tres maneras utilizando el Programa EcoSim ver. 7.0 (Gotelli \& Entsminger 2004): total de roedores nocturnos, por tamaños corporales pequeños (Peromyscus, Reithrodontomys y Onychomys) y, por tamaños medianos (Neotoma, Nelsonia y Sigmodon).

El índice de Stone \& Roberts (1990) cuantifica la cantidad promedio de coocurrencias entre todas las parejas únicas de las especies en el ensamblaje (tablero de ajedrez). Si el ensamble no está estructurado al azar el índice observado es significativamente mayor que el esperado en las simulaciones (valor Cscore). Además para confirman diferencias significativas (95\% de confianza) se calculó el efecto estandarizado $(\mathrm{SES})=\left(\mathrm{S}^{2}\right.$ Observado Promedio Simulado / $\sigma^{2}$ Simulado) en donde valores mayores a dos la confirman. Las simulaciones se corrieron con sumas fijas de hileras y columnas (suponen independencia entre las especies al momento de colonizar) y el algoritmo secuencial de intercambio, realizándose 1,000 interacciones.

Por último se calculó el grado de anidamiento del ensamble, que es una medida del orden en la cual las especies se relaciona en los sitios de un sistema, en donde si está más estructurado "se jerarquiza" (menores valores de temperatura) y evalúa el orden de desaparición de una especie en un sistema (Atmar \& Patterson 1993). Esto se hizo a partir de ordenar las especies conforme a patrones jerarquizados: de mayor a menor presencia de las especies en los sitios y de mayor a menor número de sitios ocupados por las especies, con datos de presencia/ausencia en los nueve sitios y con las 15 especies registradas en los cuadros. También se realizaron corrimientos de 50 eventos al azar con el modelo nulo de Monte Carlo, con el fin de identificar las tendencias 
del ordenamiento de las especies y utilizando el programa Nestedness Calculador (Atmar \& Patterson 1993).

\section{RESULTADOS}

Se capturaron un total de 520 roedores (386 individuos y 134 recapturas). Las capturas promedio fueron de bajas a moderadas (de 21 a 39) y similares entre las cuatro épocas del año en los tres tipos de vegetación, al igual que las capturas totales por tipo de vegetación (de 63 a 117) (Tabla 2). No se encontraron diferencias significativas entre los tres tipos de vegetación $\left(\mathrm{F}_{1,11}=1.527 ; \mathrm{P}=0.2685\right)$, ni por temporada $\left(\mathrm{F}_{1,11}=1.446 ; \mathrm{P}=0.3001\right)$. La eficiencia total de trampeo fue de $5.58 \%$ de roedores (intervalo de 3.65 a $6.77 \%$ ) y fue similar en los tres tipos de vegetación $\left(\mathrm{F}_{1,11}=1.87 ; \mathrm{P}=\right.$ $0.2093) \mathrm{y}$, por temporada $\left(\mathrm{F}_{1,11}=0.9722 ; \mathrm{P}=\right.$ 0.4522 , Tabla 2).

\section{Estructura de tallas y pesos}

La distribución de frecuencias del tamaño corporal de todas las especies de roedores fue casi unimodal, presentando un intervalo de 58 a $368 \mathrm{~mm}$ (Fig. 1A). Un primer grupo correspondió principalmente a las especies del género Peromyscus, de 67 a $97 \mathrm{~mm}$ y otro a las especies de tamaños intermedios como Sigmodon, Nelsonia, Neotoma y Tamias, de 108 a $150 \mathrm{~mm}$. En ambos grupos se cumplió la

\section{TABLA 2}

Eficiencia de trampeo de los roedores en el rancho El Durangueño, Municipio de Canatlán, Durango, México. Esfuerzo de trampeo = Número de trampas por día. Eficiencia de trampeo $=$ (Número de capturas/esfuerzo de trampeo) x 100. BQP = Bosque de Encino-Pino, BPQ = Bosque Pino-Encino, PAS = Pastizal.

Efficiency of trapping of rodents in the "El Durangueño" ranch, Municipio Canatlán, Durango, México. Effort $=$ Number of traps per day. Efficiency of trapping $=($ Number of captures/effort of trapping $) \times 100 . \mathrm{BQP}=$ Oak-pine forest, $\mathrm{BPQ}=$ Pine-oak forest, PAS = Grassland.

\begin{tabular}{|c|c|c|c|c|c|c|c|}
\hline Estación & $\begin{array}{l}\text { Tipo de } \\
\text { Vegetación }\end{array}$ & $\begin{array}{l}\text { Trampas } \\
\text { por noche }\end{array}$ & Días & $\begin{array}{c}\text { Esfuerzo } \\
\text { de trampeo }\end{array}$ & Individuos & $\begin{array}{l}\text { Capturas } \\
\text { (únicas) }\end{array}$ & $\begin{array}{c}\text { Eficiencia } \\
\text { de trampeo }(\%)\end{array}$ \\
\hline \multirow[t]{5}{*}{ Primavera } & PAS & 64 & 9 & 576 & 19 & 14 & 2.43 \\
\hline & $\mathrm{BQP}$ & 64 & 9 & 576 & 58 & 51 & 8.85 \\
\hline & BPQ & 64 & 9 & 576 & 53 & 35 & 6.08 \\
\hline & Promedio & 64 & 9 & 576 & 43 & 33 & 5.79 \\
\hline & Total & 192 & 27 & 1728 & 130 & 100 & 5.79 \\
\hline \multirow[t]{5}{*}{ Verano } & PAS & 64 & 9 & 576 & 53 & 43 & 7.47 \\
\hline & $\mathrm{BQP}$ & 64 & 9 & 576 & 51 & 41 & 7.12 \\
\hline & BPQ & 64 & 9 & 576 & 37 & 33 & 5.73 \\
\hline & Promedio & 64 & 9 & 576 & 47 & 39 & 6.77 \\
\hline & Total & 192 & 27 & 1728 & 141 & 117 & 6.77 \\
\hline \multirow[t]{5}{*}{ Otoño } & PAS & 64 & 9 & 576 & 31 & 27 & 4.69 \\
\hline & BQP & 64 & 9 & 576 & 69 & 44 & 7.64 \\
\hline & BPQ & 64 & 9 & 576 & 62 & 35 & 6.08 \\
\hline & Promedio & 64 & 9 & 576 & 54 & 35 & 6.13 \\
\hline & Total & 192 & 27 & 1728 & 162 & 106 & 6.13 \\
\hline \multirow[t]{6}{*}{ Invierno } & PAS & 64 & 9 & 576 & 31 & 26 & 4.51 \\
\hline & $\mathrm{BQP}$ & 64 & 9 & 576 & 37 & 25 & 4.34 \\
\hline & BPQ & 64 & 9 & 576 & 19 & 12 & 2.08 \\
\hline & Promedio & 64 & 9 & 576 & 29 & 21 & 3.65 \\
\hline & Total & 192 & 27 & 1728 & 87 & 63 & 3.65 \\
\hline & Total general & 768 & 108 & 6912 & 520 & 386 & 5.58 \\
\hline
\end{tabular}


regla de Hutchinson, ya que la proporción entre la talla más grande y la más pequeña fue de 1.5 y 1.4 , respectivamente. En contraste, para el peso corporal el espectro de la distribución fue amplio, con valores de 9 a 1,361 g (Fig. 1B), encontrándose gran diferencia entre la especie más pequeña (Onychomys torridus Coues, 1874) y la más grande (Sciurus niger Linnaeus, 1758). La distribución presentó un pico con cinco especies de peso similar. Los mayores pesos correspondieron a intervalos de 17 a $24 \mathrm{~g}$ (género Peromyscus), mientras que dos grupos más tuvieron hasta tres especies, las pequeñas (Reithrodontomys) y las intermedias (Sigmodon y Tamias).

Composición taxonómica, afinidad biogeográfica y gremio trófico

Se encontraron 18 especies de roedores (Tabla $3)$, pertenecientes a 10 géneros y 2 familias (Muridae y Sciuridae), siendo capturadas 15 en las trampas. Algunas especies estuvieron solo en un tipo de vegetación (Tabla 3), tres en pastizales (Sigmodon ochrognathus V.
Bailey, 1902, Reithrodontomys megalotis Baird, 1858 y Onychomys torridus Coues, 1874, dos en bosque de encino-pino (Oryzomys sp. y Neotoma lepida Thomas, 1983) y una en bosque de pino-encino (Nelsonia neotomodon Merriam, 1897). Tres especies de ardillas no fueron monitoreadas por ser de gran tamaño, pero se observaron o fueron capturadas en trampas tomahawk con un nuevo registro para Durango, la ardilla amarilla (Sciurus niger Linnaeus, 1758) principalmente asociada a árboles de gran tamaño (Pinus leiophylla Schlltdl. \& Cham. y encinares), la ardilla techalote (Sciurus nayaritensis Allen, 1889) y el ardillón (Spermophilus variegatus Erxleben, 1777) principalmente en zonas rocosas.

Las especies tienen afinidad neártica (44 $\%)$, neotropical (44\%) o compartida (11\%, Tabla 3), indicando que es una zona transicional. La mayoría de las especies son herbívoras (38.9\%), algunas granívoras (22.2 $\%)$, omnívoras $(22.2 \%)$, insectívoras $(5.6 \%)$ y frugívoras $(11.1 \%)$, de acuerdo a Ceballos y Navarro (1991).
(A)

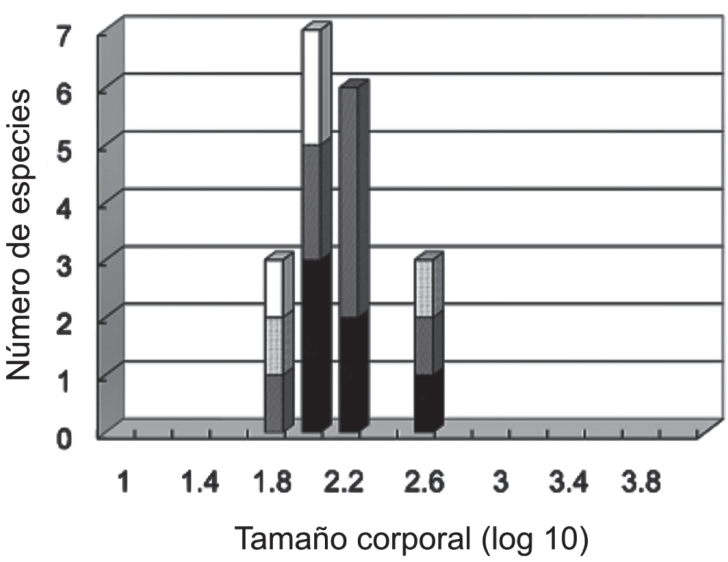

- Sierra Madre Occidental a Desierto $\square$ Grandes Planicies

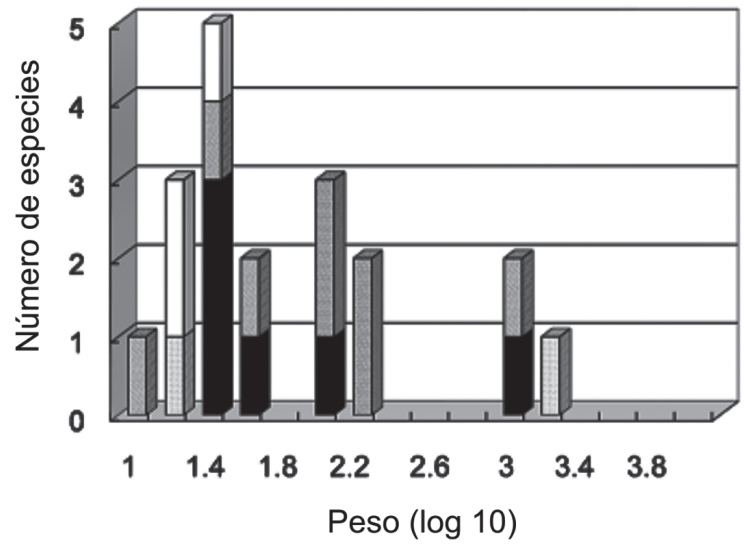

Sierra Madre Occidental $\square$ Desierto a Grandes Planicies

\section{$\square$ Amplia Distribución}

Fig. 1: Distribución de frecuencias del tamaño corporal $(\mathrm{mm})$ y del peso corporal $(\mathrm{g})$ de las especies de roedores de un bosque templado en el rancho "El Durangueño", Municipio de Canartlán, Durango, México por bioma.

Rodent body size $(\mathrm{mm})$ and body mass size (g) frequency distributions of the temperate forest in the "El Durangueño" ranch, Municipio Canatlán, Durango, México by biome. 


\section{Conservación}

Dos especies están en categoría de riesgo, la rata Nelsonia neotomodon Merriam, 1897 (protección especial y endémica de México) y la rata cambalachera (Neotoma lepida), que es rara y amenazada; esta última solo se encontró en un área rocosa con pendiente de bosques de pino-encino y en bosque de encino-pino. El techalote (Sciurus nayaritensis Allen, 1889) es una ardilla endémica de la Sierra Madre, mientras que el chichimoco (Tamias dorsalis Baird, 1855) es de distribución restringida a las partes altas de esta sierra, por lo que se sugiere que estas especies se incluyan dentro de las categorías de protección especial (SEMARNAT 2001) o vulnerable (de la IUCN).

\section{Distribución de abundancias}

Se presentaron pocas especies dominantes por tipo de vegetación, dos en pastizal (Sigmodon leucotis V. Bailey, 1902 y S. ochrognathus V. Bailey, 1902) y una en los bosques mixtos (Peromyscus truei (Shufeldt, 1885), Tabla 4).

La distribución de las especies de roedores fue variable (Tabla 4), encontrándose especies ampliamente distribuidas como Peromyscus truei (Shufeldt, 1885), Reithrodontomys montanus (Baird, 1855), P. maniculatus (Wagner, 1845) y Sigmodon leucotis V. Bailey, 1902 (ocho, siete y seis sitios; respectivamente). Otras estuvieron restringidas a un tipo de vegetación, como son Sigmodon ochrognathus V. Bailey, 1902,

TABLA 3

Composición, distribución y gremio trófico de los roedores en el rancho "El Durangueño", Municipio de Canatlán, Durango, México. Fr = Frugívoro, G = Granívoro, H = Herbívoro, O = Omnívoro, $\mathrm{I}=$ Insectívoro. Con asterisco las especies que no fueron parte del monitoreo. $\mathrm{BQP}=$ Bosque Encino-Pino, BPQ = Bosque Pino-Encino, PAS = Pastizal .

Composition, distribution and trophic guild of rodents in the "El Durangueño" ranch, Municipio Canatlán, Durango, México. $\mathrm{Fr}=$ Frugivorous, $\mathrm{G}=$ Granivorous, $\mathrm{H}=$ Herbivorous, $\mathrm{O}=$ Omnivorous, $\mathrm{I}=$ Insectivorous. An asterisk identifies the species that were not a part of the monitoring. $\mathrm{BQP}=$ Oak-pine forest, $\mathrm{BPQ}=$ Pine-oak forest, $\mathrm{PAS}=\mathrm{Grassland}$.

\begin{tabular}{|c|c|c|c|c|c|}
\hline Especies & PAS & BQP & BPQ & Afinidad Biogeográfica & Gremio Trófico \\
\hline \multicolumn{6}{|l|}{ Orden Rodentia } \\
\hline \multicolumn{6}{|l|}{ Familia Muridae } \\
\hline Peromyscus truei (Shufeldt, 1885) & $\mathrm{X}$ & $\mathrm{X}$ & $\mathrm{X}$ & Neártica y Neotropical & $\mathrm{O}$ \\
\hline Peromyscus difficilis (J. A. Allen, 1891) & & $\mathrm{X}$ & $\mathrm{X}$ & Neártica y Neotropical & G \\
\hline Peromyscus maniculatus (Wagner, 1845) & $\mathrm{X}$ & $\mathrm{X}$ & $X$ & Neártica y Neotropical & $\mathrm{O}$ \\
\hline Peromyscus boylii (Baird, 1855) & $X$ & $X$ & & Neártica & G \\
\hline Peromyscus leucopus (Rafinesque, 1818) & $\mathrm{X}$ & $\mathrm{X}$ & $\mathrm{X}$ & Neártica y Neotropical & $\mathrm{O}$ \\
\hline Neotoma mexicana Baird, 1855 & & $\mathrm{X}$ & $\mathrm{X}$ & Neártica y Neotropical & $\mathrm{H}$ \\
\hline Nelsonia neotomodon Merriam, 1897 & & & $\mathrm{X}$ & Neotropical & $\mathrm{H}$ \\
\hline Neotoma lepida Thomas, 1893 & & $\mathrm{X}$ & & Neártica & $\mathrm{H}$ \\
\hline Sigmodon leucotis V. Bailey, 1902 & $\mathrm{X}$ & $X$ & $\mathrm{X}$ & Neártica y Neotropical & $\mathrm{H}$ \\
\hline Sigmodon ochrognathus V. Bailey, 1902 & $\mathrm{X}$ & & & Neártica & $\mathrm{H}$ \\
\hline Onychomys arenicola Mearns, 1896 & & $\mathrm{X}$ & $\mathrm{X}$ & Neártica & I \\
\hline Onychomys torridus Coues, 1874 & $\mathrm{X}$ & & & Neártica & I \\
\hline Reithrodontomys megalotis Baird, 1855 & $\mathrm{X}$ & & & Neártica & G \\
\hline Reithrodontomys montanus (Baird, 1855) & $\mathrm{X}$ & $\mathrm{X}$ & $\mathrm{X}$ & Neártica & $\mathrm{G}$ \\
\hline \multicolumn{6}{|l|}{ Familia Sciuridae } \\
\hline Tamias dorsalis Baird, 1855 & & $X$ & $\mathrm{X}$ & Neártica y Neotropical & Fr \\
\hline Spermophilus variegates (Erxleben, 1777)* & & $X$ & $\mathrm{X}$ & Neártica y Neotropical & $\mathrm{H}$ \\
\hline Sciurus nayaritensis Allen, 1889* & & $\mathrm{X}$ & $\mathrm{X}$ & Neotropical & $\mathrm{H}$ \\
\hline Sciurus niger Linnaeus, 1758* & & $X$ & $\mathrm{X}$ & Neártica & $\mathrm{H}$ \\
\hline 18 especies & 10 & 14 & 13 & & \\
\hline
\end{tabular}


Reithrodontomys megalotis Baird, 1855 y Onychomys torridus Coues, 1874 (pastizal), Neotoma lepida Thomas, 1893 (encino-pino) y Nelsonia neotomodon Merriam, 1897 (pinoencino). La amplitud del nicho espacial fue mayor en Reithrodontomys montanus (Baird, 1855), Peromyscus maniculatus (Wagner, 1845) y P. truei (Shufeldt, 1885) (recíproco de Simpson de 5.3, 5.2 y 4.7 ; respectivamente). El ensamble de roedores presentó un modelo de distribución de abundancias log-normal (Fig. 2 ), es decir, tuvo pocas especies con densidades altas y muchas de bajas densidades.

\section{Parámetros del ensamble de roedores}

La riqueza de especies de roedores en todos los sitios fue alta, con 18 especies presentes en los bosques templados estudiados de la SMOcc de Durango, pero para los análisis solo se consideraron las especies capturadas en las trampas, es decir, 15 especies. Sin embargo, se detectó una riqueza local de baja a moderada en los diferentes sitios, con un intervalo de 3 a 8 especies anuales y de 1.5 a 4 por estación (Tabla 5, Tabla 6). No se encontraron diferencias estadísticas significativas entre los sitios $\left(\right.$ ANOVA $F_{1,35}=1.269, \mathrm{P}=0.3001$, Tabla 5).

Por otro lado, se encontraron diferencias estadísticas en los parámetros, diversidad, equitabilidad, dominancia y densidad de roedores entre los sitios (Figs. 3). La diversidad de roedores fue alta en general, ya que en la mayoría de los casos fue superior a uno en el total anual (promedios de 0.54 a 1.65 ) y por temporada fueron menores (promedios de 0.28 a 1.2) (ANOVA $\left.F_{1,35}=2.724, \mathrm{P}=0.02432\right)$. Se observaron diferencias significativas entre un bosque de pino-encino (Casas) y tres sitios, dos pastizales (Campo de Verano, $\mathrm{Q}_{1,16}=4.948 ; \mathrm{P}$

\section{TABLA 4}

Capturas de roedores y amplitud del nicho espacial (Recíproco del Índice de Simpson, Krebs 1999) en el rancho El Durangueño, Municipio de Canatlán, Durango, México, en los sitios monitoreados. Se resaltan en negritas los mayores valores registrados. Frec $=$ Frecuencia. Pastizal, $1=$ Campo de Verano, $2=$ Fresada, 3 = La Pista; Bosque de encino-pino, $1=$ El Aire, $2=$ La Pila, $3=$ Nogales;

Bosque de pino-encino, 1 = Casas, 2 = Kosovo, 3 = La Costosa

Captures of rodents and amplitude of the spatial niche (Reciprocal of the Index of Simpson; Krebs 1999) in the "El Durangueño" ranch, Municipio Canatlán, Durango, México, in the sampling quadrats. The highest values recorded are shown in bold. Frec $=$ Frecuency. Grassland, $1=$ Campo de Verano, $2=$ Fresada, $3=$ La Pista; Bosque de encino-pino, $1=$ El Aire, 2 = La Pila, 3 = Nogales; Bosque de pino-encino, 1 = Casas, $2=$ Kosovo, 3 = La Costosa

\begin{tabular}{|c|c|c|c|c|c|c|c|c|c|c|c|c|}
\hline \multirow[t]{2}{*}{ Especies } & \multicolumn{3}{|c|}{ Pastizal } & \multicolumn{3}{|c|}{ Bosque de encino-pino } & \multicolumn{3}{|c|}{ Bosque de pino-encino } & \multirow[t]{2}{*}{ Total } & \multirow[t]{2}{*}{ Frec } & \multirow[t]{2}{*}{ Nicho } \\
\hline & $\overline{1}$ & 2 & $\overline{3}$ & 1 & 2 & 3 & 1 & 2 & 3 & & & \\
\hline Peromyscus truei & 3 & 2 & & 24 & 48 & 4 & 11 & 22 & 24 & 138 & 8 & 4.66 \\
\hline Peromyscus difficilis & & & & 3 & 4 & & & 3 & 6 & 16 & 4 & 3.66 \\
\hline Peromyscus maniculatus & 2 & 2 & 3 & 2 & 8 & 7 & & 8 & & 32 & 7 & 5.17 \\
\hline Peromyscus boylii & & & 1 & & & 8 & & & & 9 & 2 & 1.25 \\
\hline Peromyscus leucopus & & & 1 & & 1 & 1 & 1 & & & 4 & 4 & 4 \\
\hline Neotoma mexicana & & & & & 1 & & & & & 1 & 1 & 1 \\
\hline Neotoma lepida & & & & & 2 & & & & & 2 & 1 & 1 \\
\hline Nelsonia neotomodon & & & & & & & & 2 & & 2 & 1 & 1 \\
\hline Sigmodon leucotis & 6 & 19 & 12 & 1 & & & & 2 & 1 & 41 & 6 & 3.07 \\
\hline Sigmodon ocrognathus & 10 & 6 & 5 & & & & & & & 21 & 3 & 2.74 \\
\hline Onychomys arenicola & & & & & 2 & 1 & & & 1 & 4 & 3 & 2.67 \\
\hline Onychomys torridus & & 3 & & & & & & & & 3 & 1 & 1 \\
\hline Reithrodontomys megalotis & 2 & 2 & 3 & & & & & & & 7 & 3 & 2.88 \\
\hline Reithrodontomys montanus & 6 & 6 & 6 & 2 & 1 & & 1 & 1 & 2 & 25 & 8 & 5.25 \\
\hline Tamias dorsalis & & & & 1 & & 5 & & & 4 & 10 & 3 & 2.38 \\
\hline Total de individuos & 29 & 40 & 31 & 33 & 67 & 26 & 13 & 38 & 38 & 315 & & \\
\hline
\end{tabular}


$=0.03702$ y Pista, $\left.\mathrm{Q}_{1,16}=5.414 ; \mathrm{P}=0.01708\right)$ $\mathrm{y}$ un bosque de encino-pino (Nogales, $\mathrm{Q}_{1,16}=$ 4.509; $\mathrm{P}=0.07367$; Fig. 3A).

La equitatibilidad varió entre los sitios (anual de 0.49 a 0.91 y mensual de 0.40 a 0.95), siendo más homogénea en los tres pastizales y en un sitio de bosque de encinopino (Nogales), con mayores valores (0.8), mientras que no lo fue para los otros bosques mixtos. Se detectaron diferencias estadísticas entre dos sitios (pastizal del Campo de Verano y bosque de pino-encino del cuadro Casas, Fig. 3B). La dominancia fue baja en general, excepto en un pinar (0.73), que fue significativamente diferente a dos pastizales (Campo de Verano y Pista) y a un encinar (Fig. 3C).

La densidad varió de 16 a 84 ind ha- ${ }^{-1}$ en el año y un promedio estacional de 8 a 21.5 ind ha $^{-1}$ (ANOVA F $\left.1,35=3.186, \mathrm{P}=0.01121\right)$. Se encontraron diferencias significativas entre un bosque de encino-pino (La Pila) y dos pastizales (Campo de Verano, $\mathrm{Q}_{1,16}=4.838 ; \mathrm{P}=0.04413$ ) y La Pista, $\left.\mathrm{Q}_{1,16}=5.123 ; \mathrm{P}=0.02779\right) \mathrm{y}$ dos bosques de pino-encino (Casas, $\mathrm{Q}_{1,16}=5.123$; $\mathrm{P}$ $=0.02779$ y La Costosa, $\mathrm{Q}_{1,16}=6.451 ; \mathrm{P}=$ 0.002829; Fig. 3D). Para la biomasa de roedores no se encontraron diferencias significativas por sitio $\left(\mathrm{F}_{1,35}=1.708 ; \mathrm{P}=0.1422\right)$.
Cabe resaltar que dos sitios se diferenciaron del resto, el bosque de encino-pino denominado "La Pila" por su alta densidad, siendo un sitio que presentó vegetación secundaria y un bosque de pino-encino (Casas), por su alta dominancia y ser un sitio más expuesto a la actividad humana.

Similitud de las especies de roedores entre los sitios

Se presentaron tres grupos de acuerdo a la similitud de las abundancias de las especies por sitio (Fig. 4): a) el bosque de encino-pino (La Pila), que se separa por contener las mayores densidades y corresponden a especies de alimentación generalista u oportunista (género Peromyscus), b) los pastizales, que se agruparon de acuerdo a la presencia de especies herbívoras o granívoras principalmente, mismas que son típicas de estos hábitats, tales como Sigmodon y Reithrodonthomys y a las especies de amplia distribución y oportunistas como Peromyscus y, c) grupo que incluye los bosques mixtos de pino-encino y de encinopino, en los que "Casas" y "Nogales" están más cercanos a sitios con presencia o actividad humana moderada.

\section{Log normal}

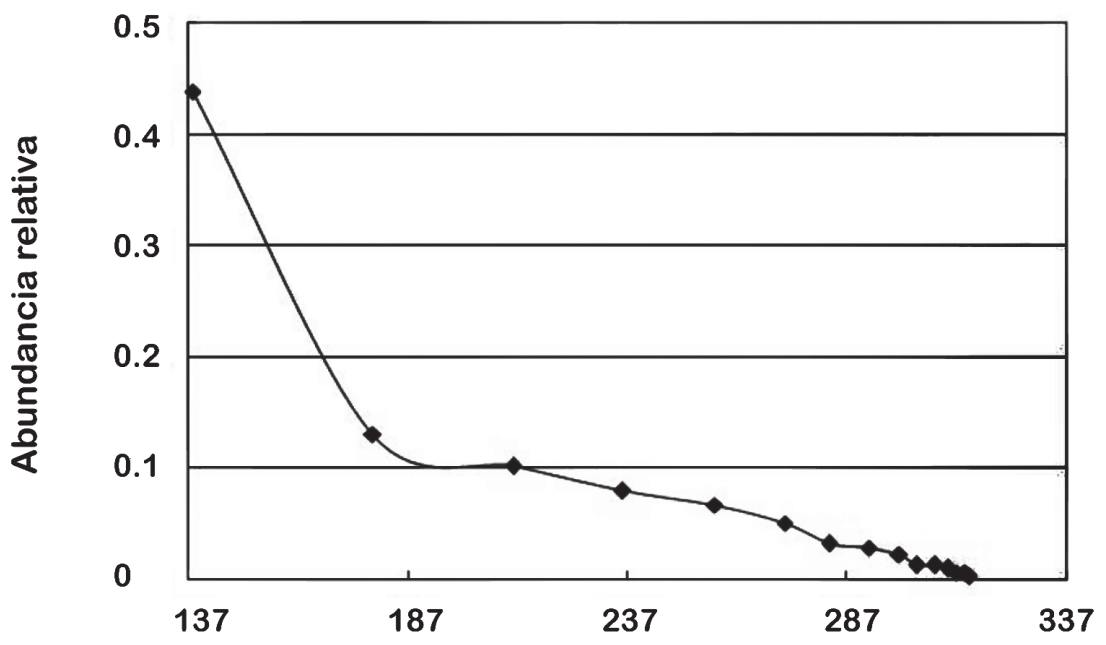

Frecuencias acumuladas

Fig. 2: Modelo de distribución de abundancias del ensamble de roedores en el rancho El Durangueño, Municipio de Canatlán, Durango, México.

Distribution model of abundances in the assemble species of rodent in the "El Durangueño" ranch, Municipio Canatlán, Durango, México. 


\section{Modelos}

El modelo nulo mostró una estructura al azar con respecto a la presencia de todas las especies de roedores nocturnos en los sitios. Sin embargo, fue distinta del azar al agrupar las especies por distintos tamaños corporales, pues la segregación espacial ocurre de acuerdo con los diferentes gremios, principalmente en cuanto al alimento y el uso espacial. Para el índice de coocurrencia (C-score) solo en el caso de los roedores de tamaño mediano fue no azaroso (108 a $150 \mathrm{~mm}$ de los géneros Neotoma, Nelsonia y Sigmodon), el valor observado fue mayor al esperado (C-Score observado $=2.3>$ C-Score esperado $=1.87$, efecto estandarizado $=2.10$ ), mientras que para los otros grupos de roedores pequeños (67 a 97 mm de los géneros Peromyscus, Onychomys y Reithrodontomys) fue lo contrario (C-Score observado $=3.31<\mathrm{C}$-Score esperado $=3.3$, efecto estandarizado $=0.06)$ y el patrón fue al azar.
El grado de anidamiento considerando todos los sitios fue de $36.29^{\circ}$, lo que indica un grado de estructura del ensamble moderado, considerando la escala de 1 a 100 grados (de mayor a menor estructura), donde los valores bajos indican una mayor estructuración (Fig. $5)$. Las tres especies más susceptibles a desaparecer de acuerdo a este estimador son, en orden decreciente, Neotoma mexicana, Neotoma lepida y Nelsonia neotomodon, y las menos susceptibles de desaparecer son Reithrodontomys montanus, Peromyscus truei y $P$. maniculatus. Estas especies corresponden a las de distribución más restringida en el sitio, ya que naturalmente están en bajas densidades (Ceballos y Oliva 2005). Sin embargo, los cálculos de probabilidad de 1,000 corrimientos mediante la simulación de Monte-Carlo, no permitieron encontrar diferencias estadísticas en la matriz $\left(\mathrm{P}\left(\mathrm{T}<36.29^{\circ}\right)=9.76 \mathrm{e}^{02}\right)$, obteniéndose un promedio de $46.4^{\circ} \pm 7.8^{\circ}$, por lo que el patrón del ensamble de roedores fue al azar o sin anidamiento.

TABLA 5

Parámetros del ensamble de especies de roedores en los sitios de muestreo en el rancho El Durangueño, Municipio de Canatlán, Durango, México. RE = riqueza específica, H' = diversidad (índice de Shannon-Wiener; Krebs 1999), E = equitabilidad, Dom = dominancia, Den = densidad $\left(\right.$ individuos $\left.\mathrm{ha}^{-1}\right)$, Peso $=$ peso de los roedores $(\mathrm{g}), \mathrm{P}=$ probabilidad, $\mathrm{F}=$ valor de Fisher (ANOVA) y $\mathrm{H}$ = estadístico $\mathrm{H}$ (*Kruskal-Wallis).

Parameters of the rodent species assembleage in the sampling sites in the "El Durangueño" ranch, Municipio Canatlán, Durango, México. RE = species richness, H' = diversity (index of Shannon-Wiener, Krebs 1999), E = equitabilidad, Dom = dominance, Den = density $\left(\right.$ individual $\left.\mathrm{ha}^{-1}\right)$, Peso = weight $(\mathrm{g}), \mathrm{P}=$ probability, $\mathrm{F}=$ value of Fisher $($ ANOVA) and, $\mathrm{H}=$ statistical $\mathrm{H}$ (*Kruskal-Wallis).

\begin{tabular}{|c|c|c|c|c|c|c|c|}
\hline Tipo de vegetación & Sitio & RE & $\mathrm{H}^{\prime}$ & $\mathrm{E}$ & Dom & Den & $\overline{\text { Peso }(\mathrm{g})}$ \\
\hline \multirow[t]{4}{*}{ Pastizal } & Campo de Verano & 6 & 1.62 & 0.91 & 0.23 & 36.25 & 915 \\
\hline & Fresada & 7 & 1.57 & 0.80 & 0.28 & 50 & 1073 \\
\hline & Pista & 7 & 1.65 & 0.85 & 0.23 & 38.75 & 1239 \\
\hline & Promedio & 6.67 & 1.61 & 0.85 & 0.25 & 41.67 & 1075.67 \\
\hline \multirow[t]{4}{*}{ B. Encino-pino } & El Aire & 6 & 1.00 & 0.56 & 0.55 & 41.25 & 692 \\
\hline & La Pila & 8 & 1.06 & 0.51 & 0.53 & 83.75 & 1188 \\
\hline & Nogales & 6 & 1.57 & 0.88 & 0.23 & 32.50 & 621 \\
\hline & Promedio & 6.67 & 1.21 & 0.65 & 0.44 & 52.50 & 833.67 \\
\hline \multirow[t]{6}{*}{ B. Pino-encino } & Casas & 3 & 0.54 & 0.49 & 0.73 & 16.25 & 269 \\
\hline & Kosovo & 6 & 1.25 & 0.70 & 0.39 & 47.50 & 1173 \\
\hline & La Costosa & 6 & 1.17 & 0.65 & 0.44 & 47.50 & 1166 \\
\hline & Promedio & 5 & 0.98 & 0.61 & 0.52 & 37.08 & 869.33 \\
\hline & $\mathrm{Fo} \mathrm{H}{ }^{*}$ & 1.269 & 2.72 & $18.44 *$ & $17.8 *$ & 3.186 & 1.708 \\
\hline & $\mathrm{P}$ & 0.3001 & 0.02432 & 0.018 & 0.0186 & 0.0112 & 0.142 \\
\hline
\end{tabular}




\section{TABLA 6}

Parámetros del ensamble de roedores en los sitios de muestreo por épocas en el rancho El Durangueño, Municipio de Canatlán, Durango, México. RE = riqueza específica, H' = diversidad (índice de Shannon-Wiener), E = equitabilidad, Dom = dominancia, Den = densidad (individuos ha $\left.{ }^{1}\right)$ y Peso $=$ peso $(\mathrm{g})$. Pastizal, 1 = Campo de Verano, 2 = Fresada, 3 = La Pista; bosque de encinopino, $1=$ El Aire, $2=$ La Pila, $3=$ Nogales; bosque de pino-encino, $1=\mathrm{Casas}, 2=\mathrm{Kosovo}, 3=\mathrm{La}$

\section{Costosa.}

Parameters of rodent assemble in the sampling sites by seasons in the "El Durangueño" ranch, Municipio Canatlán, Durango, México. RE = species richness, $\mathrm{H}^{\prime}=$ diversity (index of Shannon-Wiener), $\mathrm{E}=$ eveness, Dom = dominance, Den = density (individual ha $\left.{ }^{-1}\right)$, Peso = weight $(\mathrm{g}), \mathrm{P}=$ probability, $\mathrm{F}=$ value of Fisher and, $\mathrm{H}=$ statistical $\mathrm{H}$. Grassland, $1=$ Campo de Verano, 2 = Fresada, 3 = La Pista; oak-pine forest, $1=$ El Aire, $2=$ La Pila, 3 = Nogales; pine-oak forest, $1=$ Casas, $2=$ Kosovo, $3=$ La Costosa .

\begin{tabular}{|c|c|c|c|c|c|c|c|c|c|c|}
\hline \multirow[t]{2}{*}{ Parámetro } & \multirow[b]{2}{*}{ Época } & \multicolumn{3}{|c|}{ Pastizal } & \multicolumn{3}{|c|}{ Bosque de encino-pino } & \multicolumn{3}{|c|}{ Bosque de pino-encino } \\
\hline & & 1 & 2 & 3 & 1 & 2 & 3 & 1 & 2 & 3 \\
\hline \multirow[t]{4}{*}{ RE } & Primavera & 3 & 2 & 4 & 3 & 5 & 3 & 2 & 6 & 4 \\
\hline & Verano & 5 & 6 & 3 & 5 & 4 & 3 & 2 & 4 & 5 \\
\hline & Otoño & 2 & 2 & 4 & 2 & 5 & 4 & 1 & 3 & 3 \\
\hline & Invierno & 4 & 4 & 4 & 2 & 2 & 3 & 1 & 1 & 3 \\
\hline Promedio & & 3.5 & 3.5 & 3.8 & 3 & 4 & 3.2 & 1.5 & 3.5 & 3.8 \\
\hline Total Anual & & 6 & 7 & 7 & 6 & 8 & 6 & 3 & 6 & 6 \\
\hline \multirow[t]{4}{*}{$\mathrm{H}^{\prime}$} & Primavera & 1.10 & 0.69 & 1.32 & 0.76 & 0.54 & 1.00 & 0.69 & 1.43 & 1.03 \\
\hline & Verano & 1.52 & 1.51 & 0.97 & 1.23 & 0.82 & 0.96 & 0.41 & 0.94 & 1.04 \\
\hline & Otoño & 0.67 & 0.56 & 1.22 & 0.30 & 1.05 & 1.24 & 0.00 & 0.60 & 0.83 \\
\hline & Invierno & 1.22 & 1.09 & 1.33 & 0.38 & 0.69 & 1.01 & 0.00 & 0.00 & 0.87 \\
\hline Promedio & & 1.130 & 0.963 & 1.208 & 0.669 & 0.775 & 1.052 & 0.276 & 0.743 & 0.942 \\
\hline Total Anual & & 1.623 & 1.566 & 1.653 & 1.001 & 1.059 & 1.572 & 0.536 & 1.251 & 1.165 \\
\hline \multirow[t]{4}{*}{$\mathrm{E}$} & Primavera & 1.00 & 1.00 & 0.95 & 0.69 & 0.33 & 0.91 & 1.00 & 0.80 & 0.75 \\
\hline & Verano & 0.94 & 0.84 & 0.88 & 0.77 & 0.59 & 0.87 & 0.59 & 0.68 & 0.64 \\
\hline & Otoño & 0.97 & 0.81 & 0.88 & 0.44 & 0.65 & 0.89 & 0.00 & 0.55 & 0.76 \\
\hline & Invierno & 0.88 & 0.79 & 0.96 & 0.54 & 0.99 & 0.92 & 0.00 & 0.00 & 0.79 \\
\hline Promedio & & 0.95 & 0.86 & 0.92 & 0.61 & 0.64 & 0.90 & 0.40 & 0.51 & 0.73 \\
\hline Total Anual & & 0.91 & 0.80 & 0.85 & 0.56 & 0.51 & 0.88 & 0.49 & 0.70 & 0.65 \\
\hline \multirow[t]{4}{*}{ Dom } & Primavera & 0.33 & 0.50 & 0.28 & 0.57 & 0.78 & 0.39 & 0.50 & 0.30 & 0.45 \\
\hline & Verano & 0.24 & 0.27 & 0.41 & 0.39 & 0.57 & 0.43 & 0.76 & 0.52 & 0.50 \\
\hline & Otoño & 0.52 & 0.63 & 0.33 & 0.83 & 0.47 & 0.32 & 1.00 & 0.69 & 0.52 \\
\hline & Invierno & 0.36 & 0.42 & 0.28 & 0.78 & 0.50 & 0.39 & 1.00 & 1.00 & 0.50 \\
\hline Promedio & & 0.36 & 0.45 & 0.32 & 0.64 & 0.58 & 0.38 & 0.81 & 0.63 & 0.49 \\
\hline Total Anual & & 0.22 & 0.29 & 0.23 & 0.55 & 0.53 & 0.23 & 0.73 & 0.39 & 0.44 \\
\hline \multirow[t]{4}{*}{ Den } & Primavera & 3 & 4 & 8 & 11 & 33 & 7 & 2 & 22 & 11 \\
\hline & Verano & 12 & 21 & 9 & 12 & 22 & 7 & 7 & 10 & 16 \\
\hline & Otoño & 10 & 8 & 9 & 11 & 20 & 12 & 7 & 11 & 16 \\
\hline & Invierno & 10 & 10 & 6 & 8 & 11 & 6 & 2 & 4 & 6 \\
\hline Promedio & & 8.75 & 10.75 & 8 & 10.5 & 21.5 & 8 & 4.5 & 11.75 & 12.25 \\
\hline Total Anual & & 36.25 & 50 & 38.75 & 41.25 & 83.75 & 32.5 & 16.25 & 47.5 & 47.5 \\
\hline \multirow[t]{4}{*}{ Peso (g) } & Primavera & 55 & 39 & 216 & 234 & 750 & 325 & 36 & 641 & 353 \\
\hline & Verano & 448 & 748 & 513 & 383 & 614 & 282 & 160 & 407 & 498 \\
\hline & Otoño & 613 & 626 & 510 & 231 & 603 & 275 & 145 & 376 & 504 \\
\hline & Invierno & 315 & 407 & 152 & 148 & 197 & 208 & 41 & 78 & 164 \\
\hline Promedio & & 357.75 & 455 & 347.75 & 249 & 541 & 272.5 & 95.5 & 375.5 & 379.75 \\
\hline Total Anual & & 915 & 1073 & 1239 & 692 & 1188 & 621 & 269 & 1173 & 1166 \\
\hline
\end{tabular}


(A)

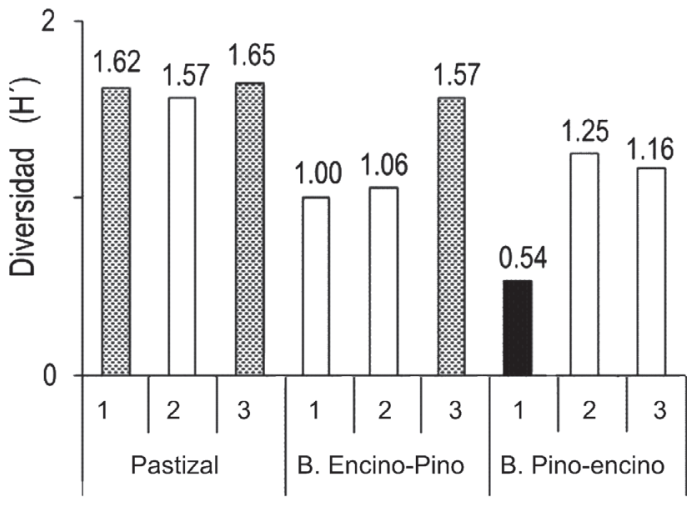

(C)

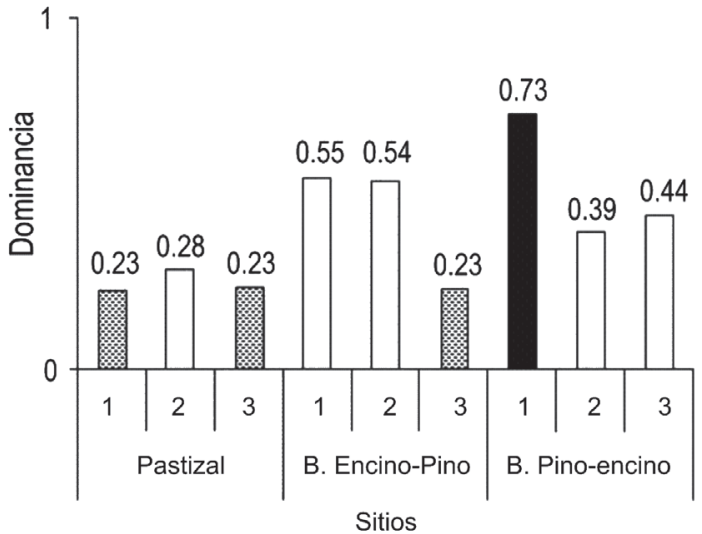

(B)

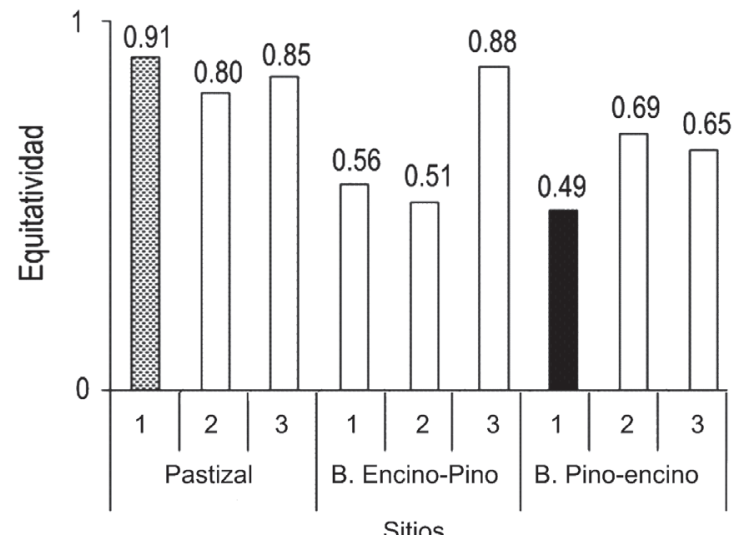

(D)

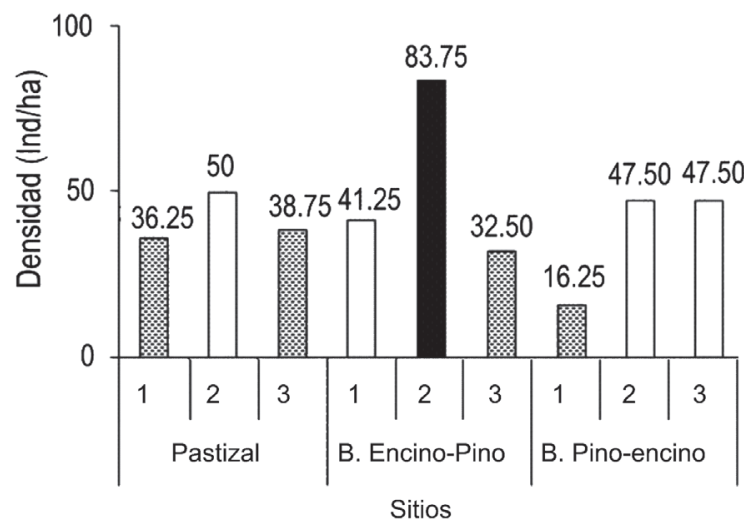

Fig. 3: Parámetros del ensamble de especies de roedores en el rancho El Durangueño, Municipio de Canatlán, Durango, México. Las barras punteadas indican diferencias estadísticas con respecto a las negras. A) Diversidad, ANOVA $\mathrm{F}_{(1,35)}=2.724, \mathrm{P}=0.02432$, Levene $=0.07$. B) Equitabilidad, Kruskal-Wallis $\mathrm{H}_{(1,35)}=18.44 ; \mathrm{P}=0.018$. C) Dominancia, Kruskal-Wallis $\mathrm{H}_{(1,35)}=17.8 ; \mathrm{P}=$ 0.0186 . D) Densidad, ANOVA $F_{(1,35)}=3.186 ; \mathrm{P}=0.012 ;$ Levene $=0.24$.

Parameters of the assemble of rodent species in the "El Durangueño" ranch, Municipio Canatlán, Durango, México. Dotted bars indicated statistical differences compared with the black bars. A) Diversity, ANOVA $\mathrm{F}_{(1,35)}=2.724, \mathrm{P}=0.02432$, Levene $=0.07$. B) Equitabilidad, Kruskal-Wallis $\mathrm{H}_{(1,35)}=18.44 ; \mathrm{P}=0.018$. C) Dominance, Kruskal-Wallis $\mathrm{H}_{(1,35)}=17.8 ; \mathrm{P}$ $=0.0186 . \mathrm{D})$ Density, ANOVA $\mathrm{F}_{(1,35)}=3.186 ; \mathrm{P}=0.012 ;$ Levene $=0.24$.

\section{DISCUSIÓN}

La estructura de los roedores en el rancho "El Durangueño" mostró una tendencia unimodal en el tamaño corporal de las especies, como se ha observado para los mamíferos de México (Torres et al. 1995, Arita \& Figueroa 1999, Ceballos et al. 2002). Los tamaños intermedios de mamíferos fueron los más comunes, como se ha reportado en mamíferos pequeños de la Reserva de la Biosfera de El Cielo en Tamaulipas, México (Castro-Arellano 2005). La proporción de tallas corporales de las especies pequeñas y medianas correspondió a la regla de Hutchinson, indicando indirectamente que las especies están repartidas en los sitios a fin de evitar la competencia (posiblemente por alimento). Mientras que la presencia de hasta de siete especies de roedores de tamaños similares del género Peromyscus, sugiere que los recursos que ofrece esta sierra son muy variados, permitiendo así su coexistencia. Lo anterior indica que existen varios elementos en el hábitat que influyen en esta característica morfológica, en donde la gran diversidad de los microambientes es debido al mosaico vegetal existente en la SMOcc (Challenger 1998). 
La distribución uniforme de pesos con respecto al tamaño corporal indica que estos bosques permiten la existencia de una gran diversidad de especies. Esto coincide con lo reportado para los mamíferos pequeños de América del Norte, en donde también hay una tendencia uniforme (Brown \& Nicoletto 1991), así como una gran variación en el peso corporal para este grupo en México (Arita \& Figueroa 1999). Lo anterior hace suponer que esta característica morfológica favorece la coexistencia interespecífica al existir diferenciación en el nicho ocupado por las especies, como un mecanismo utilizado para minimizar la competencia.

La composición de especies fue similar a la reportada para otros sitios de la SMOcc (Hernández 1996, Muñiz 1988). Sin embargo, se detectaron nuevos registros para Durango o la SMOcc, como la rata cambalachera Neotoma lepida, que estaba registrada principalmente en las zonas áridas de California y Sonora (Villa \& Cervantes 2002), la ardilla amarilla Sciurus niger, especie relicta que estaba reportada solo para el noreste de México y este de los Estados Unidos (Patterson et al. 2005) y el ratón Onychomys arenicola, principalmente de las zonas semidesérticas (Villa \& Cervantes 2003, Arita 2004). Con este estudio se corrobora que estas especies habitan las zonas de ecotono del altiplano mexicano y la SMOcc.

No se detectaron roedores heterómidos, a diferencia de lo encontrado en los bosques templados y tropicales del centro y sur de México (como Chiapas, Medellín 1992; Estado de México, Sánchez et al. 2001; Michoacán, Sánchez et al. 2005) o en las zonas áridas (Aragón \& Garza 1999, Hernández et al. 2005, González et al. 2005) donde son frecuentes. En cambio, en la zona montañosa de la SMOcc de Jalisco solo existen tres especies de heterómidos, predominan los múridos con 11 especies y cuatro sciúridos

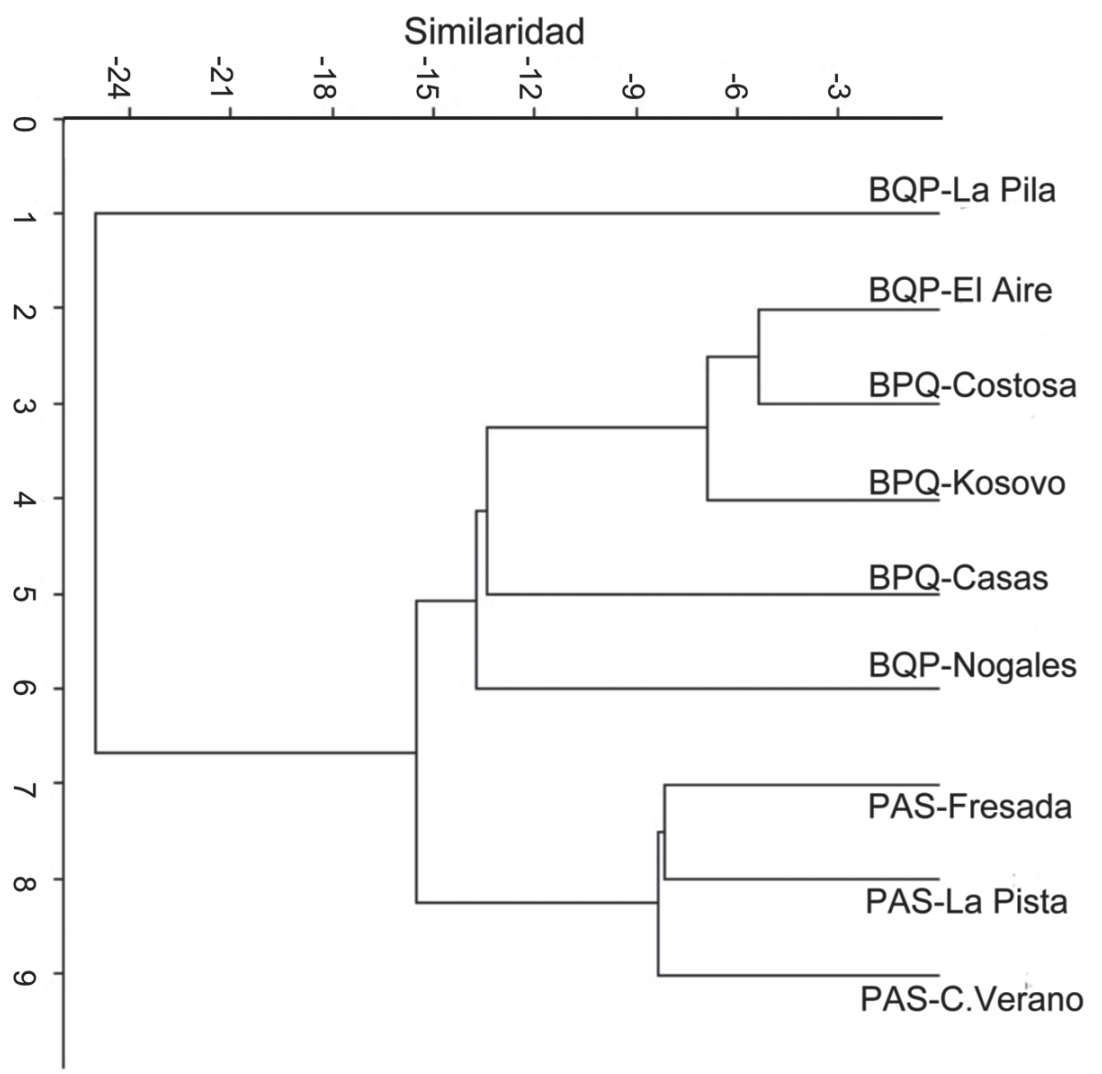

Fig. 4: Dendrograma de la similitud de la abundancia de las especies de roedores entre los sitios de muestreo del rancho El Durangueño, Municipio de Canatlán, Durango, México.

Dendrogram of the similarity of rodent species abundance among sites in "El Durangueño" ranch, Municipio Canatlán, Durango, México. 
(Guerrero et al. 1995, Íñiguez \& Santana 2005), mientras que solo existe una especie de múrido en la Reserva de la Biosfera de La Michilía (Lyomys irroratus, Hernández 1996).

El bosque de "El Durangueño" está compuesto por roedores neárticos, neotropicales o compartidos, lo que confirma que es una zona de transición, en donde confluyen tanto vegetación de montaña como del semidesierto. Es posible que los eventos históricos produjeran aislamiento o restricción en el área de distribución de las especies (Challenger 1988, Ferrusquía-Villafranca 1998, Fa \& Morales 1998), como se ha reportado en zonas montañosas de Las Filipinas, sureste de México
(Oaxaca) y Jalisco (Íñiguez \& Santana 2005) y oeste de los Estados Unidos (Brown 2001), en donde se han evidenciado sucesos como dispersión histórica, extinciones y especiación.

La amplia gama de gremios tróficos de los roedores sugiere que los recursos de este bosque son variados y disponibles, permitiendo la coexistencia interespecífica por diferencias en los hábitos alimentarios y de forrajeo. Esta estrategia se presenta principalmente en sitios con gran heterogeneidad o complejidad ambiental y con vegetación en parches, como son las zonas templadas (Cramer \& Willig 2002) y las zonas tropicales de México (Íñiguez \& Santana 2005).

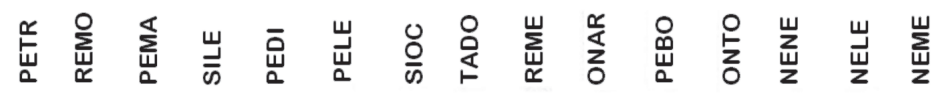

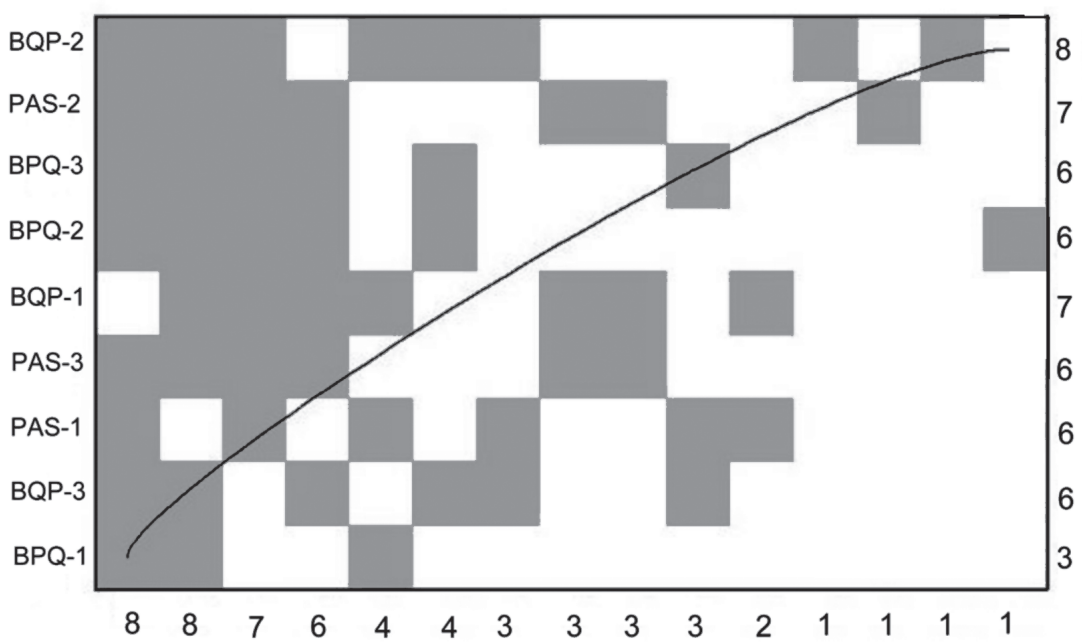

Fig 5: Patrón jerarquizado del ensamble de especies de roedores en el rancho El Durangueño, Municipio de Canatlán, Durango, México. Las especies se presentan ordenadas de menor a mayor probabilidad de extinción o desaparición de izquierda a derecha, mientras que los sitios se ordenaron de mayor a menor presencia de especies de roedores de arriba hacia abajo. Las especies son: PETR $=$ Peromyscus truei, $\mathrm{REMO}=$ Reithrodontomys montanus, $\mathrm{PEMA}=$ P. maniculatus, $\mathrm{SILE}=$ Sigmodon leucotis, PEDI $=P$. difficilis, PELE $=P$. leucopus, SIOC $=$ Sigmodon ochognathus, $\mathrm{TADO}=$ Tamias dorsalis, $\mathrm{REME}=$ Reithrodontomys megalotis, $\mathrm{PEBO}=\mathrm{P}$. boylii, $\mathrm{ONAR}=$ Onychomy arenicola, $\mathrm{ONTO}=$ O. torridus, $\mathrm{NENE}=$ Nelsonia neotomodon, NELE $=$ Neotoma lepida $\mathrm{y}$ NEME = Neotoma mexicana . Pastizal, $1=$ Campo de Verano, $2=$ Fresada, $3=$ La Pista; Bosque de encino-pino, 1 = El Aire, 2 = La Pila, 3 = Nogales; bosque de pino-encino, 1 = Casas, 2 = Kosovo, $3=$ La Costosa.

Hierarchized pattern of the assemble of rodent species in the "El Durangueño" ranch, Municipio Canatlán, Durango, Mexico. Species appear ordered from left to right corresponding to from least to highest probability of extinction or disappearance, whereas sites were order in up-down directions corresponding to greater from hisgest to smallest number of rodents species present. The species are: PETR $=$ Peromyscus truei, $\mathrm{REMO}=$ Reithrodontomys montanus, PEMA $=P$. maniculatus, $\mathrm{SILE}=$ Sigmodon leucotis, $\mathrm{PEDI}=$ P. difficilis, $\mathrm{PELE}=$ P. leucopus, $\mathrm{SIOC}=$ Sigmodon ochognathus, $\mathrm{TADO}$ $=$ Tamias dorsalis, $\mathrm{REME}=$ Reithrodontomys megalotis, $\mathrm{PEBO}=\mathrm{P}$. boylii, $\mathrm{ONAR}=$ Onychomy arenicola, $\mathrm{ONTO}=O$. torridus, NENE = Nelsonia neotomodon, NELE $=$ Neotoma lepida y NEME $=$ Neotoma mexicana . Grassland, $1=$ Campo de Verano, 2 = Fresada, 3 = Pista; oak-pine forest, 1 = El Aire, 2 = La Pila, 3 = Nogales; pine-oak forest, $1=$ Casas, 2 = Kosovo, 3 = La Costosa. 
La presencia de especies en riesgo y endemismos confirma que la SMOcc es una área de importancia para la conservación de la biodiversidad, ya que se registraron especies como Nelsonia neotomodon, Sciurus nayaritensis, Neotoma lepida y Tamias dorsalis.

La existencia de hasta dos especies dominantes por su abundancia por sitio, es un patrón general que se ha registrado en bosques tropicales de los Estados Unidos (Fleming 1975) y en bosques templados de Pensilvania (Yahner 1992), en donde una especie domina en varios hábitats y tiene fuerte influencia sobre las otras especies. Esto difiere en los bosques tropicales de Chiapas, México, en donde existen pocas especies dominantes y son distintas en cada hábitat (Medellín 1992). Las especies comunes coinciden con las de amplia distribución y de mayor amplitud de nicho espacial. La distribución lognormal de las abundancias corresponde con lo registrado para la mayoría de las comunidades con mosaicos de recursos, con especies tanto generalistas como especialistas y con comunidades distribuidas en parches (Begon et al. 1990).

Los parámetros promedio de los ensambles de roedores de los estudios desarrollados para la SMOcc, indican que la alta riqueza de especies a nivel local y regional coincide con lo reportado en otros sitios altos (Brown 2001), así como en las zonas intermedias entre la parte baja de la montaña y las cimas. En particular la alta riqueza es similar a lo estimado para otros bosques de la SMOcc, en el sureste (local de 7 a 13 especies y regional hasta 16; Muñiz 1988) y para la Reserva de la Biosfera "La Michilía" (local de 1 a 7 especies y regional hasta 11; Hernández 1996, Cautle 2007) y en Jalisco (local 7 y 19 especies regionales; Iñiguez \& Santana 2005). Sin embargo, la alta riqueza regional reportada en este estudio es la mayor registrada hasta el momento en esta sierra (18 especies), mientras que la riqueza para la Sierra Madre Occidental en Durango es de 25 y para toda esta cadena montañosa de 32. Por lo anterior, se concluye que esta provincia resguarda una gran riqueza de especies de roedores.

La diversidad de especies moderada, la equitabilidad mediana a alta y la baja dominancia en los diferentes tipos de vegetación (Tabla 5), fue similar a lo encontrado en esta sierra en la Reserva de la Biosfera "La Michilía" ( $\mathrm{H}$ ' $=0.45$ a $0.92, \mathrm{E}=$ 0.72 a $0.82, \mathrm{D}=0.50$ a 0.73 , Hernández 1996) y al sureste de Durango $\left(\mathrm{H}^{\prime}=0.98\right.$ a $2.00, \mathrm{E}=$ 0.55 a $0.87, \mathrm{D}=0.16$ a 0.52 , Muñiz 1988). La equitabilidad mayor y la dominancia baja en los pastizales demuestra que las especies están mejor repartidas. Estos valores son mayores que en otros sitios de México, en los bosques tropicales del sur $\left(\mathrm{H}^{\prime}=0.02\right.$ a $0.53, \mathrm{E}=0.08$ a 0.68 en Chiapas, Ceballos 1989, Medellín 1992, RE local $=2$ a $7, H^{\prime}=0.29-0.58$ en Veracruz y Tabasco, Sánchez et al. 2001) y similares para la Faja Volcánica Transmexicana (H' = 1.41, Michoacán, Sánchez et al. 2005).

Las densidades fueron moderadas, comparándolas con zonas aledañas a la SMOcc, la zona de ecotono entre pastizal de semidesierto y los bosques (de $54.8 \pm 28.8$ ind ha $^{-1}$ a $50.61 \pm 16.35$ ind ha ${ }^{-1}$; Sánchez 2004), los matorrales tropicales subcaducifolios de El Mezquital, Durango (144.7 \pm 47.1 ind ha $^{-1}$ a $268.0 \pm 74.3$ ind ha $^{-1}$; Sánchez 2004) y los pastizales y matorrales del Desierto Chihuahuense $5.87 \pm 0.7$ ind ha $^{-1}$ a $23.6 \pm 1.1$ ind ha ${ }^{-1}$; Hernández et al. 1995, González et al. 2005).

Los sitios de bosque que destacan por valores máximos en densidad o mínima diversidad corresponden a lugares con cierto grado de perturbación, con presencia de vegetación secundaria (bosque de encino-pino "La Pila") y el otro expuesto a la actividad humana (bosque de pino-encino "Casas") Esto difiere a lo encontrado en el bosque templado de Yellowstone y en los bosques tropicales de Brasil, donde disturbios moderados causan un incremento en la riqueza de mamíferos (Taylor 1972, Stallings 1988). Sin embargo, solo estudios de experimentación en campo en zonas perturbadas y zonas conservadas podrían confirmar estas aseveraciones.

Los roedores de tamaño mediano con un patrón espacial distinto al azaroso correspondieron a cinco especies herbívoras; mientras que el patrón al azar de las especies de tamaño pequeño, correspondió a gremios tróficos variados (omnívoras, granívoras e insectívoras), siendo estas cinco y representando el $76.6 \%$ de todos los individuos. Por lo que los patrones distintos al azar se detectaron al evaluar las especies conforme a grupos funcionales similares (gremios tróficos). 
El patrón no azaroso de los roedores medianos es similar a lo reportado en estudios realizados en un gradiente altitudinal en la Reserva de la Biosfera "El Cielo" en Tamaulipas, México (Castro-Arellano 2005). Este resultado se puede explicar de varias formas, diferencias en características del hábitat en los sitios, procesos históricos o filogenéticos y/o competencia (Brooks \& McLennan 1993, Lomolino 1999, Morris 1999). Sin embargo, información futura y precisa de estos aspectos permitirán entender con precisión cuál es la causa del patrón observado.

En contraste, la coexistencia de especies de tamaño pequeño debe existir por algún factor del hábitat que permite la permanencia de una amplia gama de gremios tróficos y posiblemente el uso diferencial los recursos existentes en los sitios. Por lo que estudios futuros detallados sobre la capacidad de dispersión, la historia evolutiva e información filogenética permitirán clarificar los mecanismos relacionados con su organización.

El grado de anidamiento del ensamble de los roedores y los subsecuentes modelos obtenidos no evidencia un patrón, si se considera a todas las especies de roedores. Los resultados sugieren que probablemente las especies que colonizaron después de los cambios durante el Pleistoceno presentaron alta competencia y exclusión en el pasado; posteriormente fueron adaptándose a nichos diversos en condiciones distintas, causados quizá por un aislamiento geográfico dada la compleja historia de los eventos ocurridos en la SMOcc (Ferruquía-Villafranca 1978, Atmar \& Patterson 1993). Es probable que estos mismos eventos produjeran la gran variedad de microambientes y en la actualidad no existan patrones a nivel del ensamble de roedores, sino que ocurra a nivel de gremios o grupos funcionales a mayor escala. Lo anterior se sustenta en que algunas especies están restringidas en su distribución, dado que sus requerimientos ecológicos son más especializados, mientras que otras son de amplia distribución y abundantes.

Se concluye que la comunidad de roedores de este bosque de la Sierra Madre Occidental tiene una alta riqueza y diversidad específica, resultado de que es una zona de transición en donde confluyen especies de las zonas neártica y neotropical, con presencia de endemismos, formando así un complejo mosaico de especies, lo que sugiere un buen estado de conservación del área.

Los resultados apoyan la hipótesis de que el ensamblaje de roedores presenta una estructura no azarosa (mayor riqueza de tallas intermedias, reparto espacial de las especies por tamaño corporal, hábitos alimentarios variados, coexistencia interespecífica de individuos pequeños por diferencias en su alimentación y segregación espacial en los de talla mediana). Esto muy probablemente es resultado de la diversidad de microambientes o heterogeneidad espacial, producto de topografía y los mosaicos vegetales que limitan la dispersión de las especies.

Por lo anterior, se sugiere realizar estudios históricos de los linajes de las especies y sobre la biogeografía histórica de esta cadena montañosa, así como incrementar los estudios ecológicos y realizar monitoreos a largo plazo en otras localidades de esta provincia, ya que son sitios de alta diversidad biológica. Este estudio representa un avance en el conocimiento sobre la organización de mamíferos pequeños en zonas templadas, que servirán de base para el uso, el manejo y la conservación de los recursos de estos bosques de la Sierra Madre Occidental.

\section{AGRADECIMIENTOS}

Este estudio forma parte del proyecto "Biodiversidad del rancho El Durangueño, Canatlán, Durango" financiado por la Comisión Nacional Forestal (CONAFOR). Se agradece al Centro de Ecología Regional, A.C. y al Posgrado en Ciencias Biológicas de la Universidad Nacional Autónoma de México por el apoyo otorgado para llevar a cabo esta investigación. Asimismo, el Instituto de Ecología, A.C. otorgó algunas facilidades para desarrollar este estudio. Así como a las personas que ayudaron para desarrollar el trabajo de campo a: Alberto Arvizu, Francisco Sánchez, Sergio Raúl Gutiérrez y Alfredo Sánchez Ríos. En especial se agradece a los Srs. Rodrigo y Alonso Ayala Grimaud, propietarios del rancho y al Sr. Issac Rafael Velázquez Escobedo, por el hospedaje y las facilidades otorgadas durante el estudio. A 
Isolda Luna Vega, Katheryn Elizabeth Stoner, Héctor Arita, Rodrigo Medellín, Zenón Cano, Jorge Servín, Gerardo Ceballos, Ella Vázquez, Miguel Briones y Victor Sánchez, por las observaciones hechas para enriquecer este trabajo y el escrito.

\section{LITERATURA CITADA}

ÁLVAREZ T \& OJ POLACO (1984) Estudio de los mamíferos capturados en la Michilía, Sureste de Durango, México. Anales de la Escuela Nacional de Ciencias Biológicas 28: 99-148.

ARAGÓN EE \& A GARZA (1999) Actualización del inventario de los mamíferos silvestres de la Reserva de la Biosfera de Mapimí. Acta Científica Potosina 14: 7-25.

ARAGÓN EE, R MUÑIZ, L HERNÁNDEZ, J LAUNDRÉ \& C LÓPEZ (en prensa) Los mamíferos de Durango. Publicaciones del Gobierno del Estado de Durango, Durango, México.

ARAGÓN EE, A GARZA, S GONZÁLEZ \& I LUNA (en prensa) Estructura y composición florística de las comunidades vegetales del Rancho "El Durangueño", en la Sierra Madre Occidental, Durango, México. Revista Mexicana de Biodiversidad.

ARITA HT (2004) Escalas y la diversidad de mamíferos de México (Proyecto P-075). Informe Técnico. Comisión Nacional para el Conocimiento y Uso de la Biodiversidad (CONABIO) y Centro de Ecología, Universidad Nacional Autónoma de México. Distrito Federal, México. 40 pp.

ARITA HT \& F FIGUEROA (1999) Geographic patterns of body-mass diversity in mexican mammals. Oikos 85: 310-319.

ATMAR W \& BD PATTERSON (1993) La medida de orden y de desorden en la distribución de la especie en hábitat hecho fragmentos. Oecología 96: 373382 .

BEGON MJ, L HARPER \& CR TOWSEND (1990) Ecology: Individuals, populations and communities. Second edition. Blackwell Scientific Publications, Cambridge, United Kingdom. 1147 pp.

BROOKS DR \& DA MCLENNAN (1993) Historical ecology: Examining phylogenetic components of community evolution. En: Ricklefs RE \& D Schluter (eds) Species diversity in ecological communities: 267-280. University of Chicago Press, Chicago, IL.

BROWN JH (2001) Mammals on mountainsides: Elevation patterns of diversity. Global Ecology and Biogeography 10: 101-109.

BROWN JH \& PF NICOLETTO (1991) Spatial scaling of species composition-body masses of NorthAmerican land mammals. The American Naturalist 138: $1478-1512$.

CANHAM CD, JS DENSLOW, WJ PLATT, JR RUKLE, TA SPIES et al. (1990) Light regimes beneath closed canopies and tree-fall gaps in temperate an tropical forest. Canadian Journal of Forest Research 20: 620-631.

CAREY AB \& ML JOHNSON (1995) Small mammals in managed, naturally young, and old-growth forest. Ecological Applications 5: 336-352.

CAREY AB, BR LIPPKE \& J SESSIONS (1999)
International ecosystem management: Managed and unmanaged forest. Wildlife Monographs 142: 1-71.

CASTRO-ARELLANO I (2005) Ecological patterns of the small mammal communities at El Cielo Biosphere Reserve, Tamaulipas, Mexico. Ph. Dissertation of Philosophy Texas A\&M University, Texas. $137 \mathrm{pp}$.

CEBALLOS G (1989) Population and community ecology of samll mammals from tropical deciduos and arroyo forest in western Mexico. Ph. D. Dissertation, University of Arizona, Tucson, Arizona.158 pp.

CEBALLOS G \& G OLIVA (eds) (2005) Los mamíferos silvestres de México. Comisión Nacional para el Conocimiento y Uso de la Biodiversidad (CONABIO)-Fondo de Cultura Económica, Distrito Federal, México. 988 pp.

CEBALLOS G \& D NAVARRO (1991) Diversity and Conservation of Mexican mammals. En: Mares MA \& DJ Schmidly (eds) Latin American Mammalogy: History, diversity and conservation: 167-198. University of Oklahoma Press, Norman, USA.

CEBALlOS G \& P RODRÍGUEZ (1993) Diversidad y conservación de los mamíferos de México: II Patrones de endemicidad. En: Medellín RA \& G Ceballos (eds) Avances en el Estudio de los mamíferos de México: 377-413, Asociación Mexicana de Mastozoología, AC. Publicación Especial No. 1, Distrito Federal, México.

CEBALLOS G, J ARROYO-CABRALES \& R MEDELLín (2002) Mamíferos de México. En: Ceballos G \& JA Simonetti (eds) Diversidad y Conservación de los Mamíferos Neotropicales: $377-$ 413. Comisión Nacional para el Conocimiento y Uso de la Biodiversidad (CONABIO)-Universidad Nacional Autónoma de México, Distrito Federal, México.

CUAUTLE LM (2007) Diversidad de roedores en la Reserva de la Biosfera La Michilía en relación con la heterogeneidad ambiental a nivel macrohábitat y microhábitat. Tesis de Maestría. Instituto de Ecología, A.C., Xalapa, México. 85 pp

CHALLENGER A (1998) Utilización y conservación de los ecosistemas terrestres de México. Pasado, presente y futuro. Comisión Nacional para el Conocimiento y Uso de la Biodiversidad (CONABIO)-Instituto de Biología, Universidad Nacional Autónoma de México y Sierra Madre, A.C., Distrito Federal, México. 847 pp.

CRAMER MJ \& MR WILLIG (2002) Habitat heterogeneity, habitat associations, and rodent species diversity in a sand-shinnery-oak landscape. Journal of Mammalogy 83: 743-753.

DIAMOND JM (1975) Assembly of species communities. En: Cody ML \& JM Diamond (eds) Ecology and evolution of communities: 342-444. Harvard University Press, Cambridge, Massachusetts.

FERRUSQUÍA-VILLAFRANCA I (1978) Distribution of Cenozoic vertebrate fauna in Middle America and problems of migration between North and South America. En: Ferrusquía-Villafranca I (ed) Conexiones Terrestres entre Norte y Sudamérica: 193-321. Boletín de la Universidad Nacional Autónoma de México, Instituto de Geología.

FERRUSQUÍA-VILLAFRANCA I (1998) Geología de México: una sinopsis. En: Ramamoothy TP, R Bye, A Lot \& J Fa (eds) Diversidad biológica de México: orígenes y distribución: 3-108. Universidad Nacional Autónoma de México, Distrito Federal, México.

FA JE \& LM MORALES (1998) Patrones de diversidad de 
mamíferos de México. En: Ramamoothy TP, R Bye, A Lot \& J Fa (eds) Diversidad biológica de México: orígenes y distribución: 199-226. Universidad Nacional Autónoma de México, Distrito Federal, México.

FLEMING TH (1975) The role of small mammals in tropical ecosystems. En: Golley FB, K Petrusewicz \& L Ryskowski (eds) Small mammals: Their productivity and population dynamics: 267-299. Cambridge University Press, Massachusetts, USA.

FOX BJ (1987) Species assembly and the evolution of community structure. Evolutionary Ecology 1: 201213.

GALINDO C (1991) Effects of habitat and food on demographic classes and population dynamics of a habitat specialist, the rock mouse. $\mathrm{Ph}$. D. Dissertation. The University of British Columbia, British Columbia, Canada. 137 pp.

GALINDO C \& CJ KREBS (1997) Habitat structure and demographic variability of a habitat specialist: The rock mouse (Peromyscus difficilis). Revista Mexicana de Mastozoología 2: 72-89.

GARCÍA E (1988) Modificaciones al sistema de clasificación de Köppen (para adaptarlo a las condiciones de la República Mexicana). Instituto de Geografía, Universidad Nacional Autónoma de México, Distrito Federal, México. 246 pp.

GARZA HA, EE ARAGÓN, E RIVERA, GD DE LEÓN, CP SÁNCHEZ et al. (2005) Biodiversidad del rancho "El Durangueño", Canatlán, Durango. Informe Técnico (III-10-CABSA-0341), Programa para el Desarrollo Forestal 2004, Comisión Nacional Forestal-Gobierno del Estado de Durango-Instituto de Ecología, AC. Durango, Durango, México. 88 pp.

GONZÁLEZ ES (1983) La vegetación de Durango. Centro Interdisciplinario de Investigación para el Desarrollo Integral Regional-Instituto Politécnico Nacional, Unidad Durango. Cuadernos de Investigación Tecnológica 1: 1-114.

GONZÁLEZ S, M GONZÁLEZ \& A CORTÉS (1993) Vegetación de la Reserva de la Biosfera "La Michilía”, Durango, México. Acta Botánica Mexicana 22: 1-104

GONZÁLEZ S, M GONZÁLEZ \& MA MÁRQUEZLINARES (2007) Vegetación y ecorregiones de Durango. Plaza y Valdés Editores-Instituto Politécnico Nacional. México, D.F. 219 pp.

GONZÁLEZ A, L HERNÁNDEZ, J LAUNDRÉ, E. ARAGÓN \& J LÓPEZ-PORTILLO (2005) Monitoreo de dos comunidades de roedores. En: Sánchez V \& R Medellín (eds) Contribución Mastozoológicas en Homenaje a Bernardo Villa: 15-26. Instituto de Biología, UNAM; Instituto de Ecología, UNAM; CONABIO, México.

GOTELLI NJ \& GR GRAVES (1996). Null models in ecology. Smithsonian Institution Press, Washington, DC. 368 pp.

GOTELLI NJ \& GL ENTSMINGER (2004). EcoSim: Null models software for ecology. (V. 7.0). Acquired Intelligence and Kesey-Bear. Jericho, Vermont, USA. URL:http://gargentsminger.com/ecosin.htm (accedido Marzo 30, 2009).

GUERRERO S, J TÉLLEZ \& R AMPARAN (1995). Los mamíferos de Jalisco: análisis zoogeográfico. Biotam 6: 1-15.

HALL ER (1981) The mammals of North America. Second edition. John Wiley \& Sons, New York, USA. 1181 pp.

HAMMER O, DA HARPER \& PD RYAN (2005) PASTPaleontological Statistics.Ver. 1.3.66 pp.
HEANEY LR (2001) Small mammal diversity along elevational gradients in the Philippines: An assessment of patterns and hypotheses. Global Ecology \& Biogeography 10: 15-39.

HERNÁNDEZ A (1996) Los pequeños mamíferos como indicadores de diversidad ambiental en la Reserva de la Biosfera La Michilía, México. Informe Técnico Final. Comité MAB/UNESCO. México, Distrito Federal.

HERNÁNDEZ L, A GONZÁLEZ, JW LAUNDRÉ, D LIGHTFOOT, EE ARAGÓN et al. (2005). Changes in rodent community structure in the Chihuahuan Desert México: Comparisons between two habitats. Journal of Arid Environments 60: 239-257.

HUTCHINSON GE (1959). Homage to Santa Rosalía; or, why are there so many kinds of animals? The American Naturalist 93: 145-159.

ÍNIIGUEZ L \& E SANTANA (2005) Análisis mastofaunístico del Estado de Jalisco. En: Sánchez V \& R Medellín (eds) Contribución mastozoológicas en homenaje a B Villa: 253-268. Instituto de Biología, UNAM; Instituto de Ecología, UNAM; Comisión Nacional para el Conocimiento y Uso de la Biodiversidad (CONABIO), México.

KOTLER BP \& JS BROWN (1988) Environmental heterogeneity and the coexistence of desert rodents. Annual Review of Ecology and Systematics 19: 281-307.

KREBS JC (1966) Demographic changes in fluctuating population of Microtus californicus. Ecological Monographs 36: 239-273.

KREBS JC (1999) Ecological methodology. Second Edition. University of British Columbia, Harper and Row, New York. 620 pp.

LERTZMAN KP, GD SUTHERLAND, A INSELBERG \& SC SAUNDERS (1996) Canopy gaps and the landscape mosaic in a coastal temperate rain forest. Ecology 77: 1254-1270.

LOMOLINO MV (1999) A species based, hierarchical model of island biogeography. En: Weiher EA \& PA Keddy (eds) Ecological assembly rules. Perspectives, advances, retreats: 272-310. Cambridge University Press, Cambridge.

MAGURRAN AE (1988) Ecological diversity and its measurement. Princeton University Press, New Jersey, USA. 179 pp

MCCAIN CHM (2004) Elevational gradients in diversity of small mammals. Ecology 86: 366-372.

MEDELLÍN RA (1992) Community ecology and conservation of mammals in Mayan tropical rainforest and abandoned agricultural fields. Ph. D. Dissertation. University of Florida, Gainesville. 332 pp.

MENA JL (2004) Diversidad y distribución de mamíferos pequeños no voladores en un gradiente altitudinal en la vertiente del pacífico de la Reserva de la Biosfera El Triunfo, Chiapas. Tesis de Maestría. Facultad de Ciencias, Universidad Nacional Autónoma de México, Distrito Federal, México. 84 pp.

MORRIS DW (1999) A haunting legacy from isoclines: Mammal coexistence and the ghost of competition. Journal of Mammalogy 80: 375-384

MUÑIZ MR (1988) Estudio ecológico de roedores colectados en el sureste del Estado de Durango. Tesis de Licenciatura. Escuela de Ciencias Biológicas, Instituto Politécnico Nacional, Distrito Federal, México. 76 pp.

NOR SMD (2001) Elevational diversity patterns of small mammals on Mount Kinabalu, Malaysia. Global Ecology and Biogeography 10: 41-62 
PATTERSON BD \& JH BROWN (1991) Regionally nested patterns of species composition in granivorous rodent assemblages. Journal of Biogeography 18: 395-402.

PATTERSON BD, DF STOTZ, S SOLARI, JW FITZPATRICK \& V PACHECO (1998) Contrasting patterns of elevational zonation for birds and mammals in the Andes of Southeastern Peru. Journal of Biogeography 25: 593-607.

PATTERSON BD, G CEBALLOS, W SECHREST, MF TOGNELLI, T BROOKS et al. (2005) Digital distribution maps of the mammals of the western hemisphere, version 2.0. Nature Serve, Arlington, Virginia, USA.

PIELOU EC (1974) Population and community ecology: Principles and methods. Gordon and Breach Science Publ., New York, USA. 424 pp.

RICKART ER (2001) Elevational diversity gradients, biogeography, and the structure of montane mammal communities in the intermountain region of North America. Global Ecology and Biogeography 10: 77-100.

RICKLEFS RE \& D SCHLUTER (1993) Species diversity: Regional and historical influences. En: Ricklefs RE \& D Schluter (eds) Species diversity in ecological communities: Historical and geographical perspectives: $350-363$. University of Chicago Press., Illinois, USA.

ROMERO ML, C SÁNCHEZ, C GARCÍA \& RD OWEN (2000) Mamíferos pequeños. Manual de técnicas de capturas, preparación, preservación y estudio. Las Prensas de Ciencias, Universidad Nacional Autónoma de México, México DF. 151 pp.

SÁNCHEZ-CORDERO V (2001) Elevational gradients of diversity for rodents and bats in Oaxaca, México. Global Ecology and Biogeography 10: 63-76.

SÁNCHEZ C, C GARCÍA \& ML ROMERO (2005) Distribución y diversidad de los roedores del Estado de Michoacán y su asociación con las Regiones Fisiográficas. En: Sánchez V \& R Medellín (eds) Contribución Mastozoológicas en Homenaje a B Villa: 451-460. Instituto de Biología, UNAM; Instituto de Ecología, UNAM; CONABIO, México.

SÁNCHEZ C, ML ROMERO, H COLÍN \& C GARCÍA (2001) Mamíferos de cuatro áreas con diferente grado de alteración en el sureste de México. Acta Zoológica Mexicana, Nueva Serie 84: 35-48.

SEMARNAT (2001) Norma Oficial Mexicana NOM-059-
SEMARNAT-2001, Protección de especies nativas de México de flora y fauna silvestres-Categorías de riesgo y especificaciones para su inclusión, exclusión o cambio-Lista de especies en riesgo. Diario Oficial de la Federación, 6 de marzo. Distrito Federal, México.

SOSA V, M ARANDA, A HERNÁNDEZ, E ARAGÓN \& J SERVÍN (1995) Inventario de los mamíferos de las Reservas Calakmul, El Cielo, Mapimí y La Michilía. Informe Técnico. Instituto de Ecología, A.C.- CONABIO. Distrito Federal, México. 56 pp.

STALLINGS JR (1988) Small mammals communities in an eastern Brazilian park. Ph. D. Dissertation. University of Florida, Gainesville, FL. 194 pp.

STEPHEN FC, C SÁNCHEZ, MK SHUMP, RW TESCA \& GRH BAKER (1975) Algunas características poblacionales demográficas de pequeños mamíferos en dos hábitats mexicanos. Anales del Instituto de Biología, Universidad Nacional Autónoma de México. Serie Zoología 46: 101-124.

STONE L \& A ROBERTS (1990) The checkerboard score and species distributions. Oecologia 85: 74-79.

STONE L, T DAYAN \& D SIMBERLOFF (2000) On desert rodents, favored status and unresolved issues: Scaling up and down regional assemblages and local communities. The American Naturalist 156: $322-328$

TAYLOR DL (1972) Some ecological implications of forest fire control in Yellowstone Park. Ecology 54: 1394-1396.

TILMAN D (1999) The ecological consequences of changes in biodiversity: A search for general principles. Ecology 80: 1455-1474.

TORRES A, C ESQUIVEL \& G CEBALLOS (1995) Diversidad y conservación de los mamíferos marinos de México. Revista Mexicana de Mastozoología 1: 22-43.

VILLA B \& F CERVANTES (2003) Los mamíferos de México. Instituto de Biología, Universidad Nacional Autónoma de México. Grupo Editorial Iberoamérica, Distrito Federal, México. 140 pp.

YAHNER RH (1992) Dynamics of a small mammal community in a fragmented forest. American Midland Naturalist 127: 381-391.

ZAR JH (1996) Biostatistical analysis. Third edition. Prentice Hall. Upper Saddle River, New Jersey, USA. 662 pp. 